\title{
Determination of a Riemannian manifold from the distance difference functions
}

\section{Lassas, Matti}

2019-04

Lassas, M \& Saksala , T 2019 , ' Determination of a Riemannian manifold from the distance

difference functions ' , Asian Journal of Mathematics , vol. 23 , no. 2 , pp. 173-199 . https://doi.org/10.4310/AJM.201

http://hdl.handle.net/10138/326781

https://doi.org/10.4310/AJM.2019.v23.n2.a1

unspecified

acceptedVersion

Downloaded from Helda, University of Helsinki institutional repository.

This is an electronic reprint of the original article.

This reprint may differ from the original in pagination and typographic detail.

Please cite the original version. 


\title{
DETERMINATION OF A RIEMANNIAN MANIFOLD FROM THE DISTANCE DIFFERENCE FUNCTIONS
}

\author{
MATTI LASSAS AND TEEMU SAKSALA
}

\begin{abstract}
Let $(N, g)$ be a Riemannian manifold with the distance function $d(x, y)$ and an open subset $M \subset N$. For $x \in M$ we denote by $D_{x}$ the distance difference function $D_{x}: F \times F \rightarrow \mathbb{R}$, given by $D_{x}\left(z_{1}, z_{2}\right)=d\left(x, z_{1}\right)-d\left(x, z_{2}\right), z_{1}, z_{2} \in F=N \backslash M$. We consider the inverse problem of determining the topological and the differentiable structure of the manifold $M$ and the metric $\left.g\right|_{M}$ on it when we are given the distance difference data, that is, the set $F$, the metric $\left.g\right|_{F}$, and the collection $\mathcal{D}(M)=\left\{D_{x} ; x \in M\right\}$. Moreover, we consider the embedded image $\mathcal{D}(M)$ of the manifold $M$, in the vector space $C(F \times F)$, as a representation of manifold $M$. The inverse problem of determining $(M, g)$ from $\mathcal{D}(M)$ arises e.g. in the study of the wave equation on $\mathbb{R} \times N$ when we observe in $F$ the waves produced by spontaneous point sources at unknown points $(t, x) \in \mathbb{R} \times M$. Then $D_{x}\left(z_{1}, z_{2}\right)$ is the difference of the times when one observes at points $z_{1}$ and $z_{2}$ the wave produced by a point source at $x$ that goes off at an unknown time. The problem has applications in hybrid inverse problems and in geophysical imaging.
\end{abstract}

Keywords: Inverse problems, distance functions, embeddings of manifolds, wave equation.

\section{Contents}

1. Introduction 2

1.1. Motivation of the problem 2

1.2. Definitions and the main result 2

1.3. Embeddings of a Riemannian manifold. 4

1.4. Earlier results and the related inverse problems 5

2. Proof of the main result 8

2.1. Extension of data 10

2.2. Manifolds $N_{1}$ and $N_{2}$ are homeomorphic. 11

2.3. Manifolds $N_{1}$ and $N_{2}$ are diffeomorphic. 13

2.4. Riemannian metrics $g_{1}$ and $\Psi_{*} g_{2}$ coincide in $N_{1}$. 16

3. Application for an inverse problem for a wave equation 25

Appendix A: Extensions of data 27

$\begin{array}{ll}\text { References } & 29\end{array}$

Date: March 29, 2018. 


\section{INTRODUCTION}

1.1. Motivation of the problem. Let us consider a body in which there spontaneously appear point sources that create propagating waves. In various applications one encounters a geometric inverse problem where we detect such waves either outside or at the boundary of the body and aim to determine the unknown wave speed inside the body. As an example of such situation, one can consider the micro-earthquakes that appear very frequently near active faults. The related inverse problem is whether the surface observations of elastic waves produced by the micro-earthquakes can be used in the geophysical imaging of Earth's subsurface $[25,58]$, that is, to determine the speed of the elastic waves in the studied volume. In this paper we consider a highly idealized version of the above inverse problem: We consider the problem on an $n$ dimensional manifold $N$ with a Riemannian metric $g$. The distance function determined by this metric tensor corresponds physically to the travel time of a wave between two points. The Riemannian distance of points $x, y \in N$ is denoted by $d(x, y)$. For simplicity we assume that the manifold $N$ is compact and has no boundary. Instead of considering measurements on boundary, we assume that the manifold contains an unknown part $M \subset N$ and the metric is known outside the set $M$. When a spontaneous point source produces a wave at some unknown point $x \in M$ at some unknown time $t \in \mathbb{R}$, the produced wave is observed at the point $z \in N \backslash M$ at time $T_{x, t}(z)=d(z, x)+t$. These observation times at two points $z_{1}, z_{2} \in N \backslash M$ determine the distance difference function

$$
D_{x}\left(z_{1}, z_{2}\right)=T_{x, t}\left(z_{1}\right)-T_{x, t}\left(z_{2}\right)=d\left(z_{1}, x\right)-d\left(z_{2}, x\right) .
$$

Physically, this function corresponds to the difference of times at $z_{1}$ and $z_{2}$ of the waves produced by the point source at $(x, t)$, see Fig 1. and Section 3. The assumption that there is a large number point sources and that we do measurements over a long time can be modeled by the assumption that we are given the set $N \backslash M$ and the family of functions

$$
\left\{D_{x} ; x \in X\right\} \subset C((N \backslash M) \times(N \backslash M)),
$$

where $X \subset M$ is either the whole manifold $M$ or its dense subset, see Remark 2.5 below.

1.2. Definitions and the main result. Let $\left(N_{1}, g_{1}\right)$ and $\left(N_{2}, g_{2}\right)$ be compact and connected Riemannian manifolds without boundary. Let $d_{j}(x, y)$ denote the Riemannian distance of points $x, y \in N_{j}, j=1,2$. Let $M_{j} \subset N_{j}$ be open sets and define closed sets $F_{j}=N_{j} \backslash M_{j}$. Suppose $F_{j}^{\text {int }} \neq \emptyset$. This is a crucial assumption and we provide a counterexample for a case $F_{j}^{i n t}=\emptyset$ in Appendix A. 


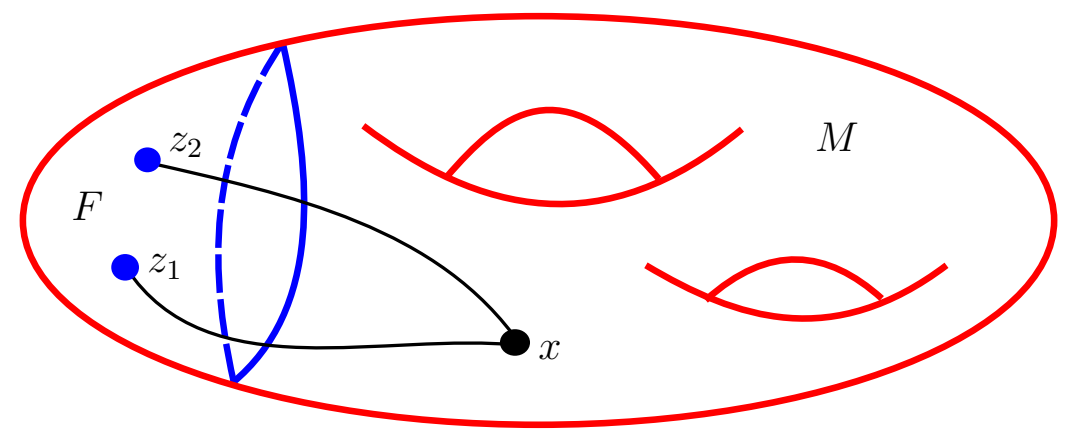

FIGURE 1. The distance difference functions are related to observation on the closed manifold $N$ that contains an unknown open subset $M$ and its known complement $F=$ $N \backslash M$. The distance difference function $D_{x}$ associated to a source point $x \in M$ has, at the observation points $z_{1}, z_{2} \in F$, the value $D_{x}\left(z_{1}, z_{2}\right)=d\left(x, z_{1}\right)-d\left(x, z_{2}\right)$. Consider the wave equation and a wave that is produced by a point source at $x$ that goes off at an unknown time and that is observed on $F$. Then the difference of the times when the wave is observed at the points $z_{1}$ and $z_{2}$ is equal to $D_{x}\left(z_{1}, z_{2}\right)$. The time difference inverse problem is to determine the topology and the isometry type of $(N, g)$ from such observations when $x$ runs over a dense subset of $M$.

Below, we assume that we know $F_{j}$ as a differentiable manifold, that is, we know the atlas of $C^{\infty}$-smooth coordinates on $F_{j}$, and the metric tensor $\left.g_{j}\right|_{F_{j}}$ on $F_{j}$, but we do not know the manifold $\left(M_{j},\left.g_{j}\right|_{M_{j}}\right)$. We assume $F_{j}$ to be a smooth manifold with smooth boundary $\partial F_{j}=\partial M_{j}$.

Definition 1.1. For $j=1,2$ and all points $x \in N_{j}$ we define the distance difference function

$$
D_{x}^{j}: F_{j} \times F_{j} \rightarrow \mathbb{R}, D_{x}^{j}\left(z_{1}, z_{2}\right):=d_{j}\left(z_{1}, x\right)-d_{j}\left(z_{2}, x\right)
$$

where $F_{j}=N_{j} \backslash M_{j}$. Recall that here $d_{j}$ is the Riemannian distance function of manifold $N_{j}$. We denote by

$$
\mathcal{D}_{j}: N_{j} \rightarrow C\left(F_{j} \times F_{j}\right), \quad \mathcal{D}_{j}(x)=D_{x}^{j}
$$

the map from a point $x$ to the corresponding distance difference function $D_{x}^{j}$. The pair $\left(F_{j},\left.g_{j}\right|_{F_{j}}\right)$ and the collection

$$
\mathcal{D}_{j}\left(M_{j}\right)=\left\{D_{x}^{j} ; x \in M_{j}\right\} \subset C\left(F_{j} \times F_{j}\right)
$$

of the distance difference functions of the points $x \in M_{j}$ is called the distance difference data for the set $M_{j}$. 
We emphasize that the above collections $\left\{D_{x}^{j}(\cdot, \cdot) ; x \in M_{j}\right\}$ are given as unindexed subsets of $C\left(F_{j} \times F_{j}\right)$, that is, for a given element $D_{x}^{j}(\cdot, \cdot)$ of this set we do not know what is the corresponding "index point" $x$.

To prove the uniqueness of this inverse problem, we assume the following:

(2) There is a diffeomorphism $\phi: F_{1} \rightarrow F_{2}$ such that $\left.\phi^{*} g_{2}\right|_{F_{2}}=\left.g_{1}\right|_{F_{1}}$,

(3) $\left\{D_{x}^{1}(\cdot, \cdot) ; x \in M_{1}\right\}=\left\{D_{y}^{2}(\phi(\cdot), \phi(\cdot)) ; y \in M_{2}\right\}$.

The following proposition states that using the small data $\mathcal{D}_{j}\left(M_{j}\right)$ we can construct the bigger data set $\mathcal{D}_{j}\left(N_{j}\right)$.

Proposition 1.2. Assume that (2)-(3) are valid. Then:

(i) The map $\phi: F_{1} \rightarrow F_{2}$, is a metric isometry, that is, $d_{1}(z, w)=$ $d_{2}(\phi(z), \phi(w))$ for all $z, w \in F_{1}$.

(ii) The collections $\mathcal{D}_{j}\left(N_{j}\right)=\left\{D_{x}^{j}(\cdot, \cdot) ; x \in N_{j}\right\} \subset C\left(F_{j} \times F_{j}\right)$ are equivalent in the following sense

$$
\left\{D_{x}^{1}(\cdot, \cdot) ; x \in N_{1}\right\}=\left\{D_{y}^{2}(\phi(\cdot), \phi(\cdot)) ; y \in N_{2}\right\} .
$$

We postpone the proof of this proposition and the other results in the introduction and give the proofs later in the paper.

The main theorem of the paper is the following:

Theorem 1.3. Let $\left(N_{1}, g_{1}\right)$ and $\left(N_{2}, g_{2}\right)$ be compact and connected Riemannian manifolds, without boundary, of dimension $n \geq 2$. Let $M_{j} \subset N_{j}$ be open sets and define closed sets $F_{j}=N_{j} \backslash M_{j}$. Assume that $F_{j}^{\text {int }} \neq \emptyset$ and that $F_{j}$ is a smooth manifold with smooth boundary $\partial F_{j}=\partial M_{j}$. Also, suppose that assumptions (2)-(3) are valid, that is, the distance difference data for sets $M_{1}$ and $M_{2}$ are equivalent. Then the manifolds $\left(N_{1}, g_{1}\right)$ and $\left(N_{2}, g_{2}\right)$ are isometric.

We prove Theorem 1.3 in Section 2. This proof is divided into 5 subsections. In the first we set notations and consider some basic facts about geodesics. In the second we prove Proposition 1.2. In the third we show that manifolds $\left(N_{j}, g_{j}\right)$ are homeomorphic. In the fourth subsection we will construct smooth atlases with which we show that manifolds $\left(N_{j}, g_{j}\right)$ are diffeomorphic. In the fifth subsection we will use techniques developed in papers [46] and [43] to prove that manifolds $\left(N_{j}, g_{j}\right)$ are isometric.

Finally, in Section 3 we give an example how the main result can be applied for an inverse source problem for a geometric wave equation.

1.3. Embeddings of a Riemannian manifold. A classical distance function representation of a Riemannian manifold is the KuratowskiWojdyslawski embedding,

$$
\mathcal{K}: x \mapsto \operatorname{dist}_{M}(x, \cdot),
$$


from $M$ to the space of continuous functions $C(M)$ on it. The mapping $\mathcal{K}: M \rightarrow C(M)$ is an isometry so that $\mathcal{K}(M)$ is an isometric representation of $M$ in a vector space.

Another important example is the Berard-Besson-Gallot representation $[10]$

$$
\mathcal{G}: M \rightarrow C\left(M \times \mathbb{R}_{+}\right), \quad \mathcal{G}(x)=\Phi_{M}(x, \cdot, \cdot)
$$

where $(x, y, t) \mapsto \Phi_{M}(x, y, t)$ is the heat kernel of the manifold $(M, g)$. The asymptotics of the heat kernel $\Phi_{M}(x, y, t)$, as $t \rightarrow 0$, determines the distance $d(x, y)$, and by endowing $C\left(M \times \mathbb{R}_{+}\right)$with a suitable topology, the image $\mathcal{G}(M) \subset C\left(M \times \mathbb{R}_{+}\right)$can be considered as an embedded image of the manifold $M$.

Theorem 1.3 implies that the set $\mathcal{D}(M)=\left\{D_{x} ; x \in M\right\}$ can be considered as an embedded image (or a representation) of the manifold $(M, g)$ in the space $C(F \times F)$ in the embedding $x \mapsto D_{x}$. Moreover, in the proof of Theorem 1.3 we show that $\left(F,\left.g\right|_{F}\right)$ and the set $\mathcal{D}(M)$ determine uniquely an atlas of differentiable coordinates and a metric tensor on $\mathcal{D}(M)$. These structures make $\mathcal{D}(M)$ a Riemannian manifold that is isometric to the original manifold $M$. Note that the metric is different than the one inherited from the inclusion $\mathcal{D}(M) \subset C(F \times F)$. Hence, $\mathcal{D}(M)$ can be considered as a representation of the manifold $M$, given in terms of the distance difference functions, and we call it the distance difference representation of the manifold of $M$ in $C(F \times F)$.

The embedding $\mathcal{D}$ is different to the above embeddings $\mathcal{K}$ and $\mathcal{G}$ in the following way that makes it important for inverse problems: With $\mathcal{D}$ one does not need to know a priori the set $M$ to consider the function space $C(F \times F)$ into which the manifold $M$ is embedded. Similar types of embedding have been also considered in the context of boundary distance functions, see Subsection 1.4.1.

In addition to the above tensor $g$ on $N$, let us consider a sequence of metric tensors $g_{k}, k \in \mathbb{Z}_{+}$on the manifold $N$ and assume that $\left.g_{k}\right|_{F}=\left.g\right|_{F}$ on $F \subset N$. We denote the Riemannian manifolds $(N \backslash$ $\left.F,\left.g_{k}\right|_{N \backslash F}\right)$, having the boundary $\partial F$, by $\left(M_{k}, g_{k}\right)$. Also, we denote by $\mathcal{D}\left(M_{k}\right) \subset C(F \times F)$ the distance difference representations of the manifolds $\left(M_{k}, g_{k}\right)$ and let $d_{H}\left(X_{1}, X_{2}\right)$ denote the Hausdorff distance of sets $X_{1}, X_{2} \subset C(F \times F)$. When $d_{H}\left(\mathcal{D}\left(M_{k}\right), \mathcal{D}(M)\right) \rightarrow 0$, as $k \rightarrow \infty$, an interesting open question is, if the manifolds $\left(M_{k}, g_{k}\right)$ converge to $(M, g)$ in the Gromov-Hausdorff topology. This type of questions have been studied for other representations e.g. in $[2,10]$, but this question is outside the context of this paper.

1.4. Earlier results and the related inverse problems. The inverse problem for the distance difference function is closely related to many other inverse problems. We review some results below: 
1.4.1. Boundary distance functions and the inverse problem for a wave equation. The reconstruction of a compact Riemannian manifold $(M, g)$ with boundary from distance information has been considered e.g. in $[27,30]$. There, one defines for $x \in M$ the boundary distance function $r_{x}: \partial M \rightarrow \mathbb{R}$ given by $r_{x}(z)=d(x, z)$. Assume that one is given the boundary $\partial M$ and the collection of boundary distance functions corresponding to all $x \in M$, that is,

$$
\partial M \text { and } \mathcal{R}(M):=\left\{r_{x} \in C(\partial M) ; x \in M\right\} .
$$

It is shown in $[27,30]$ that only knowing the boundary distance data (5) one can reconstruct the topology of $M$, the differentiable structure of $M$ (i.e., an atlas of $C^{\infty}$-smooth coordinates), and the Riemannian metric tensor $g$. Thus $\mathcal{R}(M) \subset C(\partial M)$ can be considered as an isometric copy of $M$, and the pair $(\partial M, \mathcal{R}(M))$ is called the boundary distance representation of $M$, see $[27,30]$. Similar results for non-compact manifolds are considered in [17]. Constructive solutions to determine the metric from the boundary distance functions have been developed in [14, 15] using a Riccati equation [56] for metric tensor in boundary normal coordinates and in [55] using the properties of the conformal killing tensor.

Physically speaking, functions $r_{x}$ are determined by the wave fronts of waves produced by the delta-sources $\delta_{x, 0}$ that take place at the point $x$ at time $s=0$. The distance difference functions $D_{x}^{\partial M}$ are determined by the wave fronts of waves produced by the delta-sources $\delta_{x, s}$ that take place at the point $x$ at an unknown time $s \in \mathbb{R}$.

Many hyperbolic inverse problems with time-independent metric reduce to the problem of reconstructing the isometry type of the manifold from its boundary distance functions. Indeed, in $[26,27,29,31,32$, $35,51,52$ ] it has been show that the boundary measurements for the scalar wave equation, Dirac equation, and for Maxwell's system (with isotropic scalar impedance) determine the boundary distance functions of the Riemannian metric associated to the wave velocity.

1.4.2. Hybrid inverse problems. Hybrid inverse problems are based on coupling two physical models together. In a typical setting of these problems, the first physical system is such that by controlling the boundary values of its solution, one can produce high amplitude waves, that create, e.g. due to energy absorption, a source for the second physical system. Typically, the second physical system corresponds to a hyperbolic equation with the metric

$$
d s^{2}=c(x)^{-2}\left(\left(d x^{1}\right)^{2}+\cdots+\left(d x^{n}\right)^{2}\right)
$$

corresponding to the wave speed $c(x)$. Examples of such hybrid inverse problems are encountered in thermo-acoustic and photo-acoustic imaging see e.g. $[1,5,6,7,8,59,61,60,57]$ and quantitative elastography 
$[4,22,23]$. In some cases one can use beam forming in the first physical system to make the source for the second physical system to be strongly localized, that is, to be close to a point-source, see e.g. [4, 23].

To simplify the above hybrid inverse problem, one often can do approximations by assuming that the wave speed in the second physical system is either a constant or precisely known. Usually one also assumes that the time moment when the source for the second physical system is produced is exactly known. However, when these approximations are not made, the wave speed $c(x)$ needs to be determined, too. When the source of the second physical system is produced at the given time in the whole domain $M$, the problem is studied in $[42,62]$. In the cases when the source of the second physical system are close to a point sources, one can try to determine $c(x)$ from the wavefronts that are produced by the point sources and are observed outside the domain $M$. This problem can be uniquely solved by Theorem 1.3 and we consider it in detail below in Section 3.

1.4.3. Inverse problems of micro-earthquakes. The earthquakes are produced by the accumulated elastic strain that at some time suddenly produce an earthquake. As mentioned above, the small magnitude earthquakes (e.g. the micro-earthquakes of magnitude $1<M<3$ ) appear so frequently that the surface observations of the produced elastic waves have been proposed to be used in the imaging of the Earth near active faults $[25,58]$. The so-called time-reversal techniques to study the inverse source and medium problems arising from the microseismology have been developed in [3, 16, 24].

In geophysical studies, one often approximates the elastic waves with scalar waves satisfying a wave equation. Let us also assume that the sources of such earthquakes are point-like and that one does measurements over so long time that the source-points are sufficiently dense in the studied volume. Then the inverse problem of determining the the speed of the waves in the studied volume from the surface observations of the micro-earthquakes is close to the problem studied in this paper. We note that the above assumptions are highly idealized: For example, considering the system of elastic equations would lead to a problem where travel times are determined by a Finsler metric instead of a Riemannian one. One possible way to continue the line of research conducted in this paper, would be to study, if the result of Theorem 1.3 holds on Finsler manifolds. The authors have not yet addressed this issue.

1.4.4. Broken scattering relation. If the sign in the definition of the distance difference functions is changed in (1), we come to distance sum functions

(6) $D_{x}^{+}\left(z_{1}, z_{2}\right)=d\left(z_{1}, x\right)+d\left(z_{2}, x\right), \quad x \in M, z_{1}, z_{2} \in N \backslash M$. 
This function gives the length of the broken geodesic that is the union of the shortest geodesics connecting $z_{1}$ to $x$ and the shortest geodesics connecting $x$ to $z_{2}$. Also, the gradients of $D_{x}^{+}\left(z_{1}, z_{2}\right)$ with respect to $z_{1}$ and $z_{2}$ give the velocity vectors of these geodesics. The functions (6) appear in the study of the radiative transfer equation on manifold $(N, g)$, see $[13,47,48,49,54]$. Also, the inverse problem of determining the manifold $(M, g)$ from the broken geodesic data, consisting of the initial and the final points and directions, and the total length, of the broken geodesics, has been considered in [33].

\section{Proof of the main Result}

2.0.1. Notations and basic facts on pre-geodesics. When we are concerning only one manifold, we use the shorthand notations $M, N, F$, and $g$ instead of ones with sub-indexes.

Let $(N, g)$ be a compact and connected Riemannian $n$-manifold without boundary and $n \geq 2$. We assume that $M \subset N$ is an open set of $N$ and the set $F=N \backslash M$ is a compact, $F$ contains an open set and has a smooth boundary. Suppose we know the Riemannian structure of manifold $\left(F,\left.g\right|_{F}\right)$.

We denote the Riemannian connection of the metric $g$ by $\nabla$. A unit speed geodesic of $(N, g)$ emanating from a point $(p, \xi) \in S N$ is denoted by $\gamma_{p, \xi}(t)=\exp _{p}(t \xi)$. Here, $S N=\left\{(p, \xi) \in T N ;\|\xi\|_{g}=1\right\}$. We use a short hand notation $D_{t}:=\nabla_{\dot{\gamma}_{p, \xi}(t)}$ for the covariant differentiation in the direction $\dot{\gamma}_{p, \xi}$ for vector fields along geodesic $\gamma_{p, \xi}$.

Let $p \in N$ and choose some smooth coordinates $(U, X)$ at point $p$. Denote the Christoffel symbols of connection $\nabla$ by $\Gamma_{i, j}^{k}$.

We say that a curve $\alpha\left(\left[t_{1}, t_{2}\right]\right)$ is distance minimizing if the length of this curve is equal to the distance between its end points $\alpha\left(t_{1}\right)$ and $\alpha\left(t_{2}\right)$. Also, a geodesic that is distance minimizing is called a minimizing geodesic.

We say that a curve $\alpha\left(\left[t_{1}, t_{2}\right]\right)$ is a pre-geodesic, if $\alpha(t)$ is a $C^{1}$ smooth curve such that $\dot{\alpha}(t) \neq 0$ on $t \in\left[t_{1}, t_{2}\right]$, and $\alpha\left(\left[t_{1}, t_{2}\right]\right)$ can be re-parameterized so that it becomes a geodesic.

Let us next recall some properties of the pre-geodesics. Let us consider a geodesic curve $\gamma: \mathbb{R} \rightarrow N$, satisfying in local coordinates the equation

$$
D_{t} \dot{\gamma}(t)=\frac{d^{2} \gamma^{k}}{d t^{2}}(t)+\Gamma_{i, j}^{k}(\gamma(t)) \frac{d \gamma^{i}}{d t}(t) \frac{d \gamma^{j}}{d t}(t)=0, k \in\{1, \ldots, n\} .
$$

We need the following result, often credited to Levi-Civita [38].

Lemma 2.1. Let $\kappa: \mathbb{R} \rightarrow \mathbb{R}$ be continuous and $\widetilde{\gamma}: \mathbb{R} \rightarrow N$ be a $C^{2}$-curve that satisfies the equation

(8) $\frac{d^{2} \widetilde{\gamma}^{k}}{d s^{2}}(s)+\Gamma_{i, j}^{k}(\widetilde{\gamma}(s)) \frac{d \widetilde{\gamma}^{i}}{d s}(s) \frac{d \widetilde{\gamma}^{j}}{d s}(s)=\kappa(s) \frac{d \widetilde{\gamma}^{k}}{d s}(s), k \in\{1, \ldots, n\}$. 
Then there exists a change of parameters $t: \mathbb{R} \rightarrow \mathbb{R}$ satisfying

$$
\frac{d t}{d s}(s)=\exp \left(\int_{0}^{s} \kappa(\tau) d \tau\right) .
$$

such that curve $\gamma(t):=\widetilde{\gamma}(s(t))$ solves the geodesic equation (7). Here $s(t)$ is the inverse function for $t(s)$.

Proof. The proof is a direct computation.

Let us now consider a family $\mathcal{C}$ of $C^{2}$-smooth curves defined on $U$. We denote by $\Omega$ the subbundle of $T U$ that is deternined by the velocity fields $(c, \dot{c}), c \in \mathcal{C}$. More precisely a vector $(p, v) \in T U$ satisfies $(p, v) \in \Omega$ if and only if there exist $a, t \in \mathbb{R}, c \in \mathcal{C}$ such that $(p, v)=$ $(c(t), a \dot{c}(t))$. Let $f: \Omega \rightarrow \mathbb{R}$ be a function that satisfies

$$
f(a v)=a f(v), \text { for all } a \in \mathbb{R} \text { and } v \in \Omega,
$$

i.e., it is homogeneous of degree 1 . Moreover we assume that $f$ satisfies the equation

$$
\frac{d^{2} \widetilde{\gamma}^{k}}{d s^{2}}(s)+\Gamma_{i, j}^{k}(\widetilde{\gamma}(s)) \frac{d \widetilde{\gamma}^{i}}{d s}(s) \frac{d \widetilde{\gamma}^{j}}{d s}(s)=f\left(\frac{d \widetilde{\gamma}}{d s}(s)\right) \frac{d \widetilde{\gamma}^{k}}{d s}(s),
$$

for any $\widetilde{\gamma} \in \mathcal{C}$. By Lemma 2.1 each $\widetilde{\gamma} \in \mathcal{C}$ is a pre-geodesic of connection $\nabla$.

Next we will show that also the converse result for the pre-geodesics hold. Let $\widetilde{\gamma}$ a pre-geodesic passing over the point $p$. We assume that $\widetilde{\gamma}(0)=p$. Let $s(t)$ be such a re-parametrization of $\widetilde{\gamma}$ that $\widetilde{\gamma}(s(t))=: \gamma(t)$ satisfies the geodesic equation $(7), s(0)=0$ and $\left.\frac{d}{d t} \widetilde{\gamma}(s(t))\right|_{t=0} \in S_{p} N$. Then by the chain rule it holds that

$$
\frac{d^{2} \widetilde{\gamma}^{k}}{d s^{2}}(s)+\Gamma_{i, j}^{k}(\widetilde{\gamma}(s)) \frac{d \widetilde{\gamma}^{i}}{d s}(s) \frac{d \widetilde{\gamma}^{j}}{d t}(s)=-\frac{\ddot{s}(t)}{\dot{s}(t)^{2}} \frac{d \widetilde{\gamma}^{k}}{d s}(s), k \in\{1, \ldots, n\} .
$$

Let $\Omega$ be the subbundle of $T U$ that is determined by the velocity vectorfield $(\gamma, \dot{\gamma})$. We define $f: \Omega \rightarrow \mathbb{R}$

$$
f(q, v)=\mp\|v\|_{g} \frac{\ddot{s}(t)}{\dot{s}(t)^{2}}, \text { if } \frac{v}{\|v\|_{g}}= \pm \dot{\gamma}(t), \text { for some } t \in \mathbb{R} .
$$

Thus equations (7) and (11) are equivalent in the sense that curves satisfying the latter one, for appropriate $f$, are also geodesics of metric $g$, but parametrized in a different way.

The distance function of $N$ is denoted by $d(x, y)=d_{N}(x, y)$ for $x, y \in N$. The normal vector field of $\partial M$, pointing inside $M$, is denoted by $\nu$. The boundary cut locus function is $\tau_{\partial M}: \partial M \rightarrow \mathbb{R}_{+}$,

$$
\tau_{\partial M}(z)=\sup \left\{t>0 ; d\left(\gamma_{z, \nu}(t), \partial M\right)=t\right\} .
$$

Also, we use the cut locus function of $N$ that is $\tau: T N \rightarrow \mathbb{R}_{+}$,

$$
\tau(x, \xi)=\sup \left\{t>0 ; d\left(\exp _{x}(t \xi), x\right)=t\right\} .
$$


Functions $\tau_{\partial M}(z)$ and $\tau(x, \xi)$ are continuous and satisfy the inequality (see Lemma 2.13 of [27])

$$
\tau(z, \nu(z))>\tau_{\partial M}(z), \quad z \in \partial M .
$$

2.1. Extension of data. In this subsection we prove Proposition 1.2.

Let $z_{1}, z_{2} \in \partial F=\partial M$. Then using the triangular inequality and that $d\left(z_{1}, z_{2}\right)=D_{z_{2}}\left(z_{1}, z_{2}\right)$ we see easily that

$$
d\left(z_{1}, z_{2}\right)=\sup _{x \in M} D_{x}\left(z_{1}, z_{2}\right) .
$$

Thus $\mathcal{D}(M)$ determines the distances of the boundary points, that is, the function $\left.d\right|_{\partial M \times \partial M}: \partial M \times \partial M \rightarrow \mathbb{R}$.

Lemma 2.2. Suppose that (2)-(3) are valid. Then for every $w, z \in F_{1}$ it holds that $d_{1}(w, z)=d_{2}(\phi(w), \phi(z))$.

The proof of the lemma below is very simple, but as Lemma 2.2 shows how the given data is extended to a larger data set, we present its proof. Notice that Lemma 2.2 proofs $(i)$ of the Proposition 1.2.

Proof. Let $w, z \in F_{1}$. Let $\gamma$ be a minimizing unit speed geodesic in $N_{1}$ from $z$ to $w$ and denote $S=\gamma\left(\left[0, d_{1}(w, z)\right]\right) \cap \partial M_{1}$. When $S=\emptyset$, using $\phi^{*} g_{2}=g_{1}$ we see that $d_{1}(w, z) \geq d_{2}(\phi(w), \phi(z))$.

Next, consider the case when $S \neq \emptyset$. Let $e_{1}, e_{2} \in S$ be such that $d_{1}\left(w, e_{1}\right)=\min \left\{d_{1}(w, x): x \in S\right\}$ and $d_{1}\left(z, e_{2}\right)=\min \left\{d_{1}(z, x): x \in S\right\}$. As (2)-(3) is valid, the formula (15) implies $d_{1}\left(e_{1}, e_{2}\right)=d_{2}\left(\phi\left(e_{1}\right), \phi\left(e_{2}\right)\right)$. Since $\phi: F_{1} \rightarrow F_{2}$ satisfies $\phi^{*} g_{2}=g_{1}$,

$$
\begin{aligned}
& d_{1}(w, z)=d_{1}\left(w, e_{1}\right)+d_{1}\left(e_{1}, e_{2}\right)+d_{1}\left(e_{2}, z\right) \\
& \geq d_{2}\left(\phi(w), \phi\left(e_{1}\right)\right)+d_{2}\left(\phi\left(e_{1}\right), \phi\left(e_{2}\right)\right)+d_{2}\left(\phi\left(e_{2}\right), \phi(z)\right) \\
& \geq d_{2}(\phi(w), \phi(z))
\end{aligned}
$$

The opposite inequality follows by changing the roles of $N_{1}$ and $N_{2}$.

Let us consider the case when $x \in F_{1}$. Then, Lemma 2.2 implies that for $z_{1}, z_{2} \in F_{1}$ we have

$$
\begin{aligned}
D_{x}^{1}\left(z_{1}, z_{2}\right) & =d_{1}\left(x, z_{1}\right)-d_{1}\left(x, z_{2}\right) \\
& =d_{2}\left(\phi(x), \phi\left(z_{1}\right)\right)-d_{2}\left(\phi(x), \phi\left(z_{2}\right)\right) \\
& =D_{\phi(x)}^{2}\left(\phi(x), \phi\left(z_{2}\right)\right) .
\end{aligned}
$$

Hence,

$$
\left\{D_{x}^{1}(\cdot, \cdot) ; x \in F_{1}\right\} \subset\left\{D_{y}^{2}(\phi(\cdot), \phi(\cdot)) ; y \in F_{2}\right\} .
$$

Changing roles of $N_{1}$ and $N_{2}$ and considering $\phi^{-1}: F_{2} \rightarrow F_{1}$ instead of the diffeomorphism $\phi: F_{1} \rightarrow F_{2}$, we see that in formula (17) we have the equality. This and formula (3), together with Lemma 2.2, imply Proposition 1.2.

q.e.d. 
2.2. Manifolds $N_{1}$ and $N_{2}$ are homeomorphic. To simplify the notations, we will next in our considerations omit the sub-indexes of sets $M_{1}, N_{1}$, and $F_{1}$ and just consider the sets $M, N$, and $F$.

Let $x \in N$ and define a function $D_{x}: F \times F \rightarrow \mathbb{R}$ by a formula

$$
D_{x}\left(z_{1}, z_{2}\right)=d\left(x, z_{1}\right)-d\left(x, z_{2}\right) .
$$

Let $\mathcal{D}: N \rightarrow C(F \times F)$ be given by $\mathcal{D}(x)=D_{x}$. Next, we consider the function space $C(F \times F)$ with the norm $\|f\|_{\infty}=\sup _{x, y \in F}|f(x, y)|$

Theorem 2.3. The image $\mathcal{D}(N)=\left\{D_{x} ; x \in N\right\} \subset C(F \times F)$ is a topological manifold homeomorphic to manifold $N$. Moreover, $\mathcal{D}(M)$ is homeomorphic to $M$.

Proof. The proof consists of four short steps.

Step 1 First, we will show that $\mathcal{D}$ is 2-Lipschitz and therefore continuous. Let $x, y \in N$. Using the triangular inequality we see that

$$
\begin{aligned}
& \left\|D_{x}-D_{y}\right\|_{\infty}=\sup _{z_{1}, z_{2} \in F}\left|D_{x}\left(z_{1}, z_{2}\right)-D_{y}\left(z_{1}, z_{2}\right)\right| \\
& \quad \leq \sup _{z_{1}, z_{2} \in F}\left|d\left(x, z_{1}\right)-d\left(y, z_{1}\right)\right|+\left|d\left(x, z_{2}\right)-d\left(y, z_{2}\right)\right| \\
& \quad \leq 2 d(x, y) .
\end{aligned}
$$

Step 2. Next we will show that $\mathcal{D}$ is injective. Suppose that $x, y \in N$ are such that $D_{x}=D_{y}$ and $x \neq y$. Let $q \in F^{\text {int }}$ and denote $\ell_{x}=d(q, x)$ and $\ell_{y}=d(q, y)$. Next, without loss of generality, we assume that $\ell_{x} \leq \ell_{y}$. Also, let $\eta \in S_{q} N$ be such that $\gamma_{q, \eta}\left(\left[0, \ell_{x}\right]\right)$ is a minimizing geodesic from $q$ to $x$. Let $s_{1}>0$ be such that $s_{1}<\min \left(\ell_{x}, \ell_{y}\right)$ and $\gamma_{q, \eta}\left(\left[0, s_{1}\right]\right) \subset F^{i n t}$. Consider a point $p=\gamma_{q, \eta}(s)$ with $s \in\left[0, s_{1}\right]$. Then we see that

$$
\begin{aligned}
(d(q, p)+d(p, y))-d(q, y) & =d(q, p)+D_{y}(p, q) \\
& =d(q, p)+D_{x}(p, q) \\
& =(d(q, p)+d(p, x))-d(q, x)=0
\end{aligned}
$$

and hence $p$ is on a minimizing geodesic from $q$ to $y$.

Let us consider a minimizing geodesic $\alpha$ from $p$ to $y$ with the length $\ell_{y}-s$. Then the union of the geodesics $\gamma_{q, \eta}([0, s])$ and $\alpha$ is a distance minimizing curve from $q$ to $y$ and thus this union is a geodesic. This implies that $\alpha$ is a continuation of the geodesics $\gamma_{q, \eta}([0, s])$ and hence $y=\gamma_{q, \eta}\left(\ell_{y}\right)$. Summarizing, $\gamma_{q, \eta}\left(\left[0, \ell_{x}\right]\right)$ and $\gamma_{q, \eta}\left(\left[0, \ell_{y}\right]\right)$ are distance minimizing geodesics from $q$ to $x$ and $y$, respectively. Since $x \neq y$, we have $\ell_{x} \neq \ell_{y}$. Then, as we have assumed that $\ell_{x} \leq \ell_{y}$, we see that $\ell_{x}<\ell_{y}$.

Let $\widehat{q} \in F^{\text {int }}$ be a such point that $\widehat{q}$ is not on the curve $\gamma_{q, \eta}(\mathbb{R})$. Clearly, such a point exists as $N$ has the dimension $n \geq 2$. Let $\widehat{\ell}_{x}=$ $d(\widehat{q}, x)$ and $\widehat{\ell}_{y}=d(\widehat{q}, y)$. Also, let $\widehat{\eta} \in S_{\widehat{q}} N$ be such that $\gamma_{\widehat{q}, \widehat{\eta}}\left(\left[0, \widehat{\ell}_{x}\right]\right)$ is minimizing geodesic from $\widehat{q}$ to $x$. As above, we see that then $\gamma_{\widehat{q}, \widehat{\eta}}\left(\left[0, \widehat{\ell}_{x}\right]\right)$ 
and $\gamma_{\widehat{q}, \widehat{\eta}}\left(\left[0, \widehat{\ell}_{y}\right]\right)$ are distance minimizing geodesics from $\widehat{q}$ to $x$ and $y$, respectively. However, the geodesics $\gamma_{q, \eta}(\mathbb{R})$ and $\gamma_{\widehat{q}, \widehat{\eta}}(\mathbb{R})$ do not coincide as point sets and hence the vectors $\dot{\gamma}_{q, \eta}\left(\ell_{x}\right) \in T_{x} N$ and $\dot{\gamma}_{\widehat{q}, \widehat{\eta}}\left(\widehat{\ell}_{x}\right) \in T_{x} N$ are not parallel. Recall that $\ell_{x}<\ell_{y}$. In the case when $\widehat{\ell}_{x}<\widehat{\ell}_{y}$, let $\beta$ be the geodesic segment $\gamma_{\widehat{q}, \widehat{\eta}}\left(\left[\widehat{\ell}_{x}, \widehat{\ell}_{y}\right]\right)$ connecting $x$ to $y$. In the case when $\widehat{\ell}_{x}>\widehat{\ell}_{y}$, let $\beta$ be the geodesic segment $\gamma_{\widehat{q}, \widehat{\eta}}\left(\left[\widehat{\ell}_{y}, \widehat{\ell}_{x}\right]\right)$ connecting $x$ to $y$.

Then we see that the union of the paths $\gamma_{q, \eta}\left(\left[0, \ell_{x}\right]\right)$ and $\beta$ is a distance minimizing path from $q$ to $y$. As the vectors $\dot{\gamma}_{q, \eta}\left(\ell_{x}\right)$ and $\dot{\gamma}_{\widehat{q}, \widehat{\eta}}\left(\widehat{\ell}_{x}\right)$ are not parallel, we see that the union of these curves is not a geodesic. This is contradiction and hence there are no $x, y \in N$ such that $D_{x}=D_{y}$ and $x \neq y$. Thus, $\mathcal{D}: N \rightarrow C(F \times F)$ is an injection.

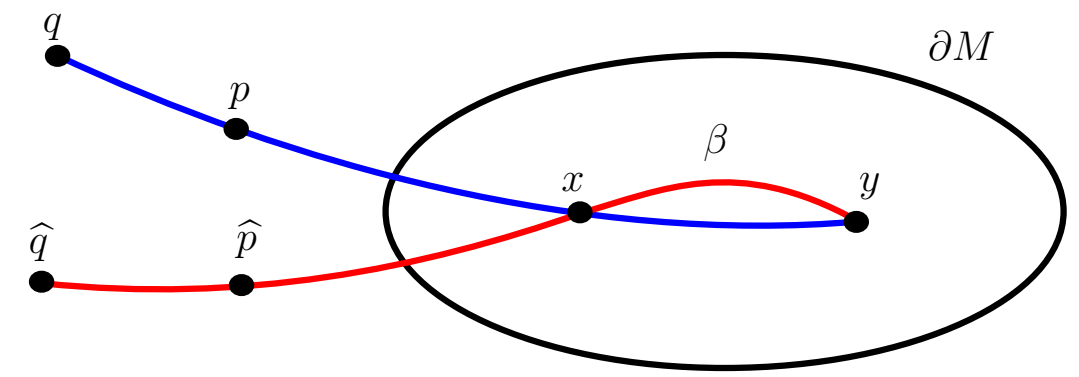

FIgURE 2. The setting in Step 2 in the proof of Theorem 2.3. We consider points $x, y \in N$ and points $p$ and $q$ such that $p$ is on a distance minimizing geodesic from $q$ to $x$. Then this geodesic can be extended to a distance minimizing geodesic from $q$ to $y$. Similarly, the point $\widehat{p}$ is on a distance minimizing geodesic from $\widehat{q}$ to $x$ and this geodesic can be extended to a distance minimizing geodesic from $\widehat{q}$ to $y$. Then the union of the (blue) geodesic from $q$ to $x$ and the (red) geodesic $\beta$ is a length minimizing curve from $q$ to $y$ that is not a geodesic.

Step 3. So far we have proved the continuity and injectivity of mapping $\mathcal{D}$. Since the domain $N$ of the mapping $\mathcal{D}$ is compact and $\left(C(F \times F),\|\cdot\|_{\infty}\right)$ is a Hausdorff space as a metric space, it holds by basic results of topology that mapping $\mathcal{D}: N \rightarrow \mathcal{D}(N)$ is a homeomorphism.

Step 4. By assumption $M \subset N$ is open and therefore mapping $\mathcal{D}$ : $M \rightarrow \mathcal{D}(M)$ is open. This proves that the mapping $\mathcal{D}: M \rightarrow \mathcal{D}(M)$ is a homeomorphism.

Define a mapping

$$
\Phi: C\left(F_{2} \times F_{2}\right) \rightarrow C\left(F_{1} \times F_{1}\right), \Phi(f)=f \circ(\phi \times \phi) .
$$

Here $f \times h: X \times X \rightarrow Y \times Y$ is defined as $(f \times h)\left(x_{1}, x_{2}\right)=\left(f\left(x_{1}\right), h\left(x_{2}\right)\right) \in$ $Y \times Y$ for mappings $f, h: X \rightarrow Y$. 
Theorem 2.4. Suppose that Riemannian manifolds $\left(N_{1}, g_{1}\right)$ and $\left(N_{2}, g_{2}\right)$ are as in section 1.2 and the assumptions of the Proposition 1.2 are valid. Let $\Phi$ be given by (19). Then the mapping

$$
\Psi:=\mathcal{D}_{1}^{-1} \circ \Phi \circ \mathcal{D}_{2}: N_{2} \rightarrow N_{1}
$$

is a homeomorphism. In addition, it holds that $\left.\Psi^{-1}\right|_{F_{1}}=\phi$.

Proof. Due the Theorem 2.3, for the first claim, we only have to prove that mapping $\Phi$ is a homeomorphism. Note that mapping $\Phi$ has an inverse mapping $h \mapsto h \circ\left(\phi^{-1} \times \phi^{-1}\right)$. Let $(x, y) \in F_{1} \times F_{1}$ and $f, h \in$ $C\left(F_{2} \times F_{2}\right)$ then it follows

$$
|(\Phi(f)-\Phi(h))(x, y)|=|f(\phi(x), \phi(y))-h(\phi(x), \phi(y))| \leq\|f-h\|_{\infty} .
$$

This proves the continuity of $\Phi$. A similar argument where $\phi$ is replaced by $\phi^{-1}$ proves that mapping $\Phi$ is a homeomorphism.

Let $x \in F_{1}$ and denote $y=\phi(x)$. Then

$$
\begin{aligned}
\Psi^{-1}(x) & =\left(\mathcal{D}_{2}^{-1} \circ \Phi^{-1} \circ \mathcal{D}_{1}\right)(x)=\mathcal{D}_{2}^{-1}\left(D_{x}^{1}\left(\phi^{-1}(\cdot) \times \phi^{-1}(\cdot)\right)\right) \\
& \stackrel{(16)}{=} \mathcal{D}_{2}^{-1}\left(D_{y}^{2}\right)=y .
\end{aligned}
$$

Remark 2.5. As the map $\mathcal{D}: M \rightarrow \mathcal{D}(M), x \mapsto D_{x}$, is a homeomorphism, we see that for a dense set $X \subset M$ we have

$$
\left.\mathcal{D}(M)=\operatorname{cl}(\mathcal{D}(X))=\operatorname{cl}\left\{D_{x} ; x \in X\right\} \subset C(F \times F)\right\},
$$

where the closure cl is taken with respect to the topology of $C(F \times F)$. This means that the distance difference functions corresponding to $x$ in a dense set $X$ determine the distance difference functions corresponding to the points in the whole set $M$.

2.3. Manifolds $N_{1}$ and $N_{2}$ are diffeomorphic. Our next goal is to construct such smooth atlases for manifolds $N_{i}$ that homeomorphism $\Psi: N_{2} \rightarrow N_{1}$ of Theorem 2.4 is a diffeomorphism.

Lemma 2.6. Let $(N, g)$ be a compact Riemannian manifold of dimension $n, x \in N$ and $\xi \in T_{x} N,\|\xi\|_{g}=1$. Let $\gamma_{x, \xi}:[0, \ell] \rightarrow N$ be a distance minimizing geodesic. Let $0<h<\ell, z=\gamma_{x, \xi}(h)$. Then there exists a neighborhood $V$ of $z$ such that the set

$$
U=\left\{\left(z_{i}\right)_{i=1}^{n} \in V^{n}: \operatorname{dim} \operatorname{span}\left(\left(F\left(z_{i}\right)-\xi\right)_{i=1}^{n}\right)=n\right\}
$$

is open and dense in $V^{n}:=V \times V \times \ldots \times V$. Here $F(q):=\frac{\left(\exp _{x}\right)^{-1}(q)}{\left\|\left(\exp _{x}\right)^{-1}(q)\right\|_{g}}, q \in$ $V$.

Moreover for every $\left(z_{i}\right)_{i=1}^{n} \in U$ there exists an open neighborhood $W$ of $x$ such that

$$
H: W \rightarrow \mathbb{R}^{n}, H(y)=\left(d\left(y, z_{i}\right)-d(y, z)\right)_{i=1}^{n}
$$

is a smooth coordinate mapping. 


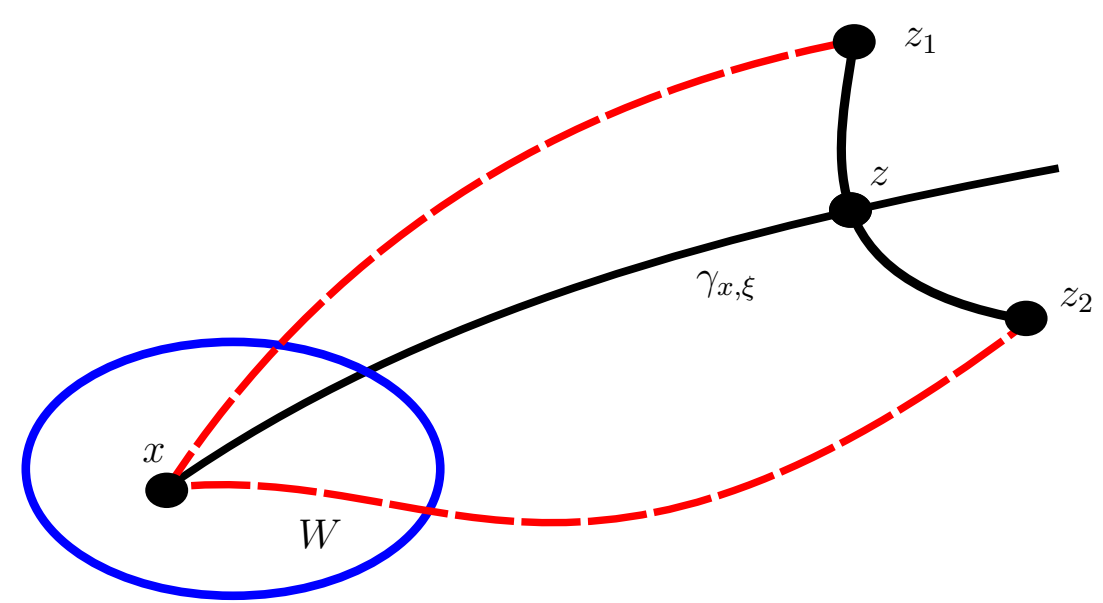

FigURE 3. A schematic picture of the coordinate system $H$.

Proof. Since the geodesic $\gamma_{x, \xi}([0, \ell])$ is distance minimizing, the geodesic segment $\gamma_{x, \xi}([0, h])$ from $x$ to $z$ has no cut points. Moreover, there exist neighborhoods $V_{x}$ and $V$ of $x$ and $z$ such that the mapping $(p, q) \mapsto d(p, q)$ is smooth on $V_{x} \times V$. As the geodesic $\gamma_{x, \xi}([0, h])$ has no cut points, the differential of $\exp _{x}$ at $v:=h \xi \in T_{x} N$ is invertible. In particularly the map $F: V \rightarrow S_{x} N$ is well defined and smooth.

Now we study the properties of the set $U$, given in (21). Consider the function

$$
T:\left(S_{x} N\right)^{n} \rightarrow \mathbb{R}, T\left(\left(v_{i}\right)_{i=1}^{n}\right)=\operatorname{det}\left(v_{1}-\xi, \ldots, v_{n}-\xi\right) .
$$

Then it holds that $\left(z_{i}\right)_{i=1}^{n} \in U$ if and only if $T\left(\left(F\left(z_{i}\right)\right)_{i=1}^{n}\right) \neq 0$. Therefore the set $U$ is open.

We define a set

$$
O:=T^{-1}(\mathbb{R} \backslash\{0\}) \subset\left(S_{x} N\right)^{n} .
$$

Then $O$ is open. Our aim is to prove that the set $O$ is also dense. We note that $\left(S_{x} N\right)^{n}$ is a real analytic manifold and the map $T$ is real analytic since, it is a polynomial. Also the constant map $0=:\left(v_{i}\right)_{i=1}^{n} \mapsto$ 0 is real analytic. By Lemma 4.3 of [20] the functions $T$ and 0 coincide in $\left(S_{x} N\right)^{n}$ if and only if they coincide in some open subset of $\left(S_{x} N\right)^{n}$. Thus to prove that $O$ is dense, it suffices to prove that there exists $\left(v_{i}\right)_{i=1}^{n} \in\left(S_{x} N\right)^{n}$ such that $T\left(\left(v_{i}\right)_{i=1}^{n} \neq 0\right.$.

To simplify the notations we assume $S_{x} N=S^{n-1} \subset \mathbb{R}^{n}$ and $\xi=e_{n}$, where $e_{1}, \ldots, e_{n}$ is the standard orthonormal basis of $\mathbb{R}^{n}$. Denote $v_{i}=$ $e_{i}, i \in\{1, \ldots, n-1\}$ and $v_{n}=\frac{v_{1}+v_{2}}{\sqrt{2}}$. Then $T\left(\left(v_{i}\right)_{i=1}^{n}\right) \neq 0$, since

$$
\operatorname{span}\left(v_{1}-\xi, \ldots, v_{n-1}-\xi, \frac{v_{1}+v_{2}}{\sqrt{2}}-\xi\right)=\operatorname{span}\left(e_{1}, \ldots, e_{n-1}, e_{n}\right)=\mathbb{R}^{n}
$$

We conclude that the set $U$ is dense in $V^{n}$, since $O \subset\left(S_{x} N\right)^{n}$ is dense and $F$ is an open map. 
Finally we will show that the mapping $H$, defined in (22), is a smooth coordinate map at some neighborhood $W$ of $x$. Choose $\left(z_{j}\right)_{j=1}^{n} \in U$. By the preparations made above, it holds that the gradients

$$
-\left.\nabla\left(d\left(\cdot, z_{i}\right)-d(\cdot, z)\right)\right|_{x}=F\left(z_{i}\right)-\xi
$$

are linearly independent. Then due to the Inverse function theorem, there exists such a neighborhood $W$ of $x$ that the function

$$
H: W \rightarrow \mathbb{R}^{n}, H(y)=\left(d\left(y, z_{i}\right)-d(y, z)\right)_{i=1}^{n}
$$

is a smooth coordinate mapping.

Next we consider the homeomorphism $\Psi: N_{2} \rightarrow N_{1}$ of Theorem 2.4.

Theorem 2.7. Suppose that Riemannian manifolds $\left(N_{1}, g_{1}\right)$ and $\left(N_{2}, g_{2}\right)$ are as in section 1.2 and Proposition 1.2 is valid. Then mapping $\Psi: N_{2} \rightarrow N_{1}$, given in formula (20), is a diffeomorphism.

Proof. Note that for any $p \in N_{2}$ and all $q, r \in F_{2}$ it holds that

$$
D_{p}^{2}(q, r)=D_{\Psi(p)}^{1}(\Psi(q), \Psi(r)) .
$$

Let $x \in N_{2}, y \in F_{2}^{\text {int }}$ and denote $\widetilde{x}=\Psi(x)$ and $\widetilde{y}=\Psi(y)$. Let $h \in$ $\left(0, d_{2}(x, y)\right)$ be such that $z:=\gamma_{x, \xi_{2}}(h) \in F_{2}^{\text {int }}$ and $\gamma_{x, \xi_{2}}\left(\left[h, d_{2}(x, y)\right]\right) \subset$ $F_{2}^{\text {int }}$, where $\gamma_{x, \xi_{2}}$ is a minimizing unit speed geodesic from $x$ to $y$ and $\widetilde{z}=\Psi(z) \in F_{1}^{\text {int }}$. Note that by the choice of $z$ it holds that it is not a cut point of $x$ on curve $\gamma_{x, \xi_{2}}$. Therefore mapping $p \mapsto D_{p}^{2}(r, q)$ is $C^{\infty}$-smooth, when $p$ is sufficiently close to $x$ and $r, q$ are sufficiently close to $z$. Since

$D_{x}^{2}(y, z)=D_{\widetilde{x}}^{1}(\widetilde{y}, \widetilde{z}), d_{2}(z, y) \geq d_{1}(\widetilde{z}, \widetilde{y})$ and $d_{2}(x, y)=d_{2}(x, z)+d_{2}(z, y)$, we deduce using the triangle inequality that

$$
d_{1}(\widetilde{x}, \widetilde{y})=d_{1}(\widetilde{x}, \widetilde{z})+d_{1}(\widetilde{z}, \widetilde{y}) .
$$

Therefore, there exists a unit speed distance minimizing geodesic $\gamma_{\widetilde{x}, \xi_{1}}$ from $\widetilde{x}$ to $\widetilde{y}$ that contains the point $\widetilde{z}$. Hence, the mapping $\widetilde{p} \mapsto D_{\widetilde{p}}^{1}(\widetilde{r}, \widetilde{q})$ is smooth, when $\widetilde{p}$ is sufficiently close to $\widetilde{x}$ and $\widetilde{r}, \widetilde{q}$ are sufficiently close to $\widetilde{z}$.

Choose a neighborhood $V_{2}$ of $z$ such that the map $F_{2}: V_{2} \rightarrow$ $S_{x} N_{2}, F_{2}(q):=\frac{\left(\exp _{x}\right)^{-1}(q)}{\left\|\left(\exp _{x}\right)^{-1}(q)\right\|_{g}}, q \in V_{2}$ is well defined. Since $\Psi$ is homeomorphism we may assume that $\Psi\left(V_{2}\right)=V_{1}$, which is a neighborhood of $\widetilde{z}$ such that the map $F_{1}: V_{1} \rightarrow S_{x} N_{1}, F_{1}(q):=\frac{\left(\exp _{x}\right)^{-1}(q)}{\left\|\left(\exp _{x}\right)^{-1}(q)\right\|_{g}}, q \in V_{1}$ is well defined.

We want to show that there exist points $\left(z_{i}\right)_{i=1}^{n} \in V_{2}$ for which the collections

$$
\left(\left(F_{2}\left(z_{i}\right)-\xi_{2}\right)\right)_{i=1}^{n} \in T_{x} N_{2} \text { and }\left(\left(F_{1}\left(\Psi\left(z_{i}\right)\right)-\xi_{1}\right)\right)_{i=1}^{n} \in T_{\widetilde{x}} N_{1}
$$

are linearly independent. Let us define

$$
U_{i}:=\left\{\left(z_{j}\right)_{j=1}^{n} \in V_{i}^{n}: \operatorname{dim} \operatorname{span}\left(\left(F_{i}\left(z_{j}\right)-\xi_{i}\right)_{j=1}^{n}\right)=n\right\}, i \in\{1,2\} .
$$


By Lemma 2.6 the sets $U_{i}$ are open and dense. Since $\Psi: N_{2} \rightarrow N_{1}$ is a homeomorphism, also the map $\Psi^{n}: N_{2}^{n} \rightarrow N_{1}^{n}$ defined by

$$
\Psi^{n}\left(\left(q_{i}\right)_{i=1}^{n}\right)=\left(\Psi\left(q_{i}\right)\right)_{i=1}^{n}
$$

is a homeomorphism. Therefore $U_{1} \cap \Psi^{n}\left(U_{2}\right)$ is open and dense in $V_{1}^{n}$. Due to the choice of vector $\xi_{1} \in S_{\widetilde{x}} N_{1}$, there exist points $\left(z_{i}\right)_{i=i}^{n} \in U_{2}$ that satisfy $\left(\Psi\left(z_{1}\right)\right)_{i=1}^{n} \in U_{1}$.

By Lemma 2.6 there exists a neighborhood $W_{2}$ of $z$ such that the map

$$
H: W_{2} \rightarrow \mathbb{R}^{n}, H(y)=\left(d_{2}\left(y, z_{i}\right)-d_{2}(y, z)\right)_{i=1}^{n}
$$

is a smooth coordinate map, $W_{1}:=\Psi\left(W_{2}\right)$ is a neighborhood of $\widetilde{x}$ and moreover the map

$$
\widetilde{H}: W_{1} \rightarrow \mathbb{R}^{n}, \widetilde{H}(y)=\left(d_{1}\left(y, \Psi\left(z_{i}\right)\right)-d_{2}(y, \widetilde{z})\right)_{i=1}^{n}
$$

is also a smooth coordinate map. We conclude that by equation (23) we have shown $H\left(W_{2}\right)=\widetilde{H}\left(W_{1}\right)$ and more importantly

$$
\widetilde{H} \circ \Psi \circ H^{-1}=I d
$$

Since the point $x \in N_{2}$ above is arbitrary and $H$ and $\widetilde{H}$ are smooth coordinate mappings for $x$ and $\widetilde{x}$, respectively, the above implies that $\Psi$ is a diffeomorphism.

2.4. Riemannian metrics $g_{1}$ and $\Psi_{*} g_{2}$ coincide in $N_{1}$. In this section we will show that manifolds $\left(N_{1}, g_{1}\right)$ and $\left(N_{2}, g_{2}\right)$ that satisfy (2)(3) are isometric.

Definition 2.8. Let $z_{1} \in F$ and $\xi \in S_{z_{1}} N$. Define a set $\sigma\left(z_{1}, \xi\right)$ by $\sigma_{N}\left(z_{1}, \xi\right):=\left\{x \in N \quad ; \quad D_{x}\left(\cdot, z_{1}\right)\right.$ is $C^{1}$-smooth in a neighborhood

$$
\text { of } \left.z_{1} \text { and }\left.\nabla D_{x}\left(\cdot, z_{1}\right)\right|_{z_{1}}=\xi\right\} \text {. }
$$

Lemma 2.9. Let $z_{1} \in F$ and $\xi \in S_{z_{1}} N$. Then it follows

$$
\sigma_{N}\left(z_{1}, \xi\right)=\gamma_{z_{1},-\xi}\left(\left\{s ; 0<s<\tau\left(z_{1},-\xi\right)\right\}\right),
$$

Roughly speaking, Lemma 2.9 means that sets $\sigma_{N}\left(z_{1}, \xi\right)$, that can be determined using data (4), are unparameterized geodesics on $N$.

Proof. First we recall that for all $x \in N$ the distance function $d(\cdot, x)$ is not smooth near $y \in N \backslash\{x\}$ if and only if point $y$ is in a cut locus of $x$. See for instance Section 9 of Chapter 5 of [56].

First, consider the case when $x \in \sigma_{N}\left(z_{1}, \xi\right)$. Then by the definition of $\sigma_{N}\left(z_{1}, \xi\right)$ the distance function $d(\cdot, x)$ is $C^{\infty}$-smooth in a neighbourhood $z_{1}$ so that $z_{1}$ is not in a cut locus of $x$, or equivalently, $x$ is not in a cut locus of $z_{1}$. Also, have that $x \neq z_{1}$. Hence, there exists an unique distance minimizing unit speed geodesic from $x$ to $z_{1}$. Since this geodesic has the velocity

$$
\left.\nabla d(\cdot, x)\right|_{z_{1}}=\left.\nabla D_{x}\left(\cdot, z_{2}\right)\right|_{z_{1}}=\xi
$$


at $z_{1}$, it follows that $x \in \gamma_{z_{1},-\xi}\left(\left\{s ; 0<s<\tau\left(z_{1},-\xi\right)\right\}\right)$.

Second, consider the case when $x \in \gamma_{z_{1},-\xi}\left(\left\{s ; 0<s<\tau\left(z_{1},-\xi\right)\right\}\right)$. Then function $D_{x}\left(\cdot, z_{1}\right)$ is smooth near $z_{1}$ and

$$
\left.\nabla D_{x}\left(\cdot, z_{1}\right)\right|_{z_{1}}=\dot{\gamma}\left(d\left(x, z_{1}\right)\right)=-\dot{\gamma}_{z_{1},-\xi}(0)=\xi .
$$

Thus, $x \in \sigma_{N}\left(z_{1}, \xi\right)$.

The Lemma 2.9 will be the key element to prove that the mapping $\Psi$ is an isometry.

Definition 2.10. Let $N$ be a smooth manifold and let $g$ and $\widetilde{g}$ be metric tensors on $N$. We say that metric tensors $g$ and $\widetilde{g}$ are geodesically equivalent, if for all geodesics $\gamma: I_{1} \rightarrow N$ of metric $g$ and $\widetilde{\gamma}: \widetilde{I}_{1} \rightarrow N$ of metric $\widetilde{g}$ there exist changes of parameters $\alpha: I_{2} \rightarrow I_{1}$ and $\widetilde{\alpha}: \widetilde{I}_{2} \rightarrow \widetilde{I}_{1}$ such that

$$
\gamma \circ \alpha \text { is a geodesic of metric } \widetilde{g}
$$

and

$$
\widetilde{\gamma} \circ \widetilde{\alpha} \text { is a geodesic of metric } g \text {. }
$$

A trivial example of two geodesically equivalent Riemannian metrics are $g$ and $c g$, where $c>0$ is a constant. Other, more interesting examples of geodesically equivalent Riemannian metrics are

(1) The Southern hemisphere of the sphere $S^{2}$ and the plane $\mathbb{R}^{2}$ and that are mapped to each other in a gnomonic projection, i.e. the great circles are mapped to straight line.

(2) Unit disc in $\mathbb{R}^{2}$ and the Beltrami-Klein model of a hyperbolic plane.

Our first goal is to show that when the distance difference data on $N_{1}$ and $N_{2}$ satisfy (2)-(3), we have that metric tensors $g_{1}$ and $\left(\Psi^{-1}\right)^{*} g_{2}$ are geodesically equivalent. By Lemma 2.9 we know all the geodesics of $N_{1}$ that exit unknown region $M_{1}$, as point sets. Next we will show that this information is enough to deduce the geodesic equivalence of $g_{1}$ and $\left(\Psi^{-1}\right)^{*} g_{2}$.

Since the mapping $\Psi$ is a diffeomorphism, it holds that each geodesic of $\left(N_{2}, g_{2}\right)$ is mapped to some smooth curve of $\left(N_{1}, g_{1}\right)$. By formula (4) and Lemma 2.9, it holds that sets $\sigma_{N}(z, \xi) \subset N_{1}$, with $z \in F_{1}$ and $\xi \in S_{z} N_{1}$, are images of geodesics of $\left(N_{2}, g_{2}\right)$ in the mapping $\Psi$. Note that the geodesic segments $\sigma_{N}(z, \xi) \subset N_{1}$ are not self-intersecting, since a cut point occurs before a geodesic stops being one-to-one.

Let $z \in F_{2}, \xi \in S_{z} N_{2}$ and $t_{2}=\tau_{2}(z,-\xi)$. Then $t \mapsto \Psi\left(\gamma_{z,-\xi}^{2}(t)\right), t \in$ $\left[0, t_{2}\right)$ is smooth and not self-intersecting curve on $N_{1}$. By Proposition 1.2 and Theorem 2.4 we have

$$
\Psi\left(\gamma_{z,-\xi}^{2}\left(\left(0, t_{2}\right)\right)\right)=\sigma_{N_{1}}\left(\Psi(z), \Psi_{*} \xi\right)=\sigma_{N_{1}}\left(\phi^{-1}(z),\left(\phi^{-1}\right)_{*} \xi\right) .
$$


Let $w=\phi^{-1}(z)$ and $\eta=\left(\phi^{-1}\right)_{*} \xi$. Then by Lemma 2.9 we have $\sigma_{N_{1}}(w, \eta)=\gamma_{w,-\eta}^{1}\left(\left\{s ; 0<s<t_{1}\right\}\right)$, where $t_{1}=\tau_{1}(w,-\eta)$. Furthermore, it is easy to see that there is a re-parametrization

$$
s:\left(0, t_{1}\right) \rightarrow\left(0, t_{2}\right) \text { such that } \gamma_{w,-\eta}^{1}(t)=\Psi\left(\gamma_{z,-\xi}^{2}(s(t))\right), t \in\left(0, t_{1}\right) .
$$

For $p \in N_{1}$, we define a collection $\mathcal{C}(p)$ of geodesics $\gamma$ of $\left(N_{1}, g_{1}\right)$ and real numbers $t_{0} \in \mathbb{R}$, given by

$$
\begin{aligned}
\mathcal{C}(p)= & \left\{\left(\gamma, t_{0}\right) ; \gamma:(a, b) \rightarrow N_{1} \text { is a geodesic of }\left(N_{1}, g_{1}\right),\right. \\
& \gamma\left(t_{0}\right)=p, \text { and there are } z \in F_{1}^{\text {int }}, \xi \in S_{z} N_{1} \\
& \text { such that } \left.\gamma((a, b))=\sigma_{N_{1}}(z, \xi)\right\} .
\end{aligned}
$$

Here $\gamma$ is given as a pair of the set $\operatorname{dom}(\gamma)=(a, b) \subset \mathbb{R},-\infty \leq a<b \leq$ $\infty$, where the mapping $\gamma$ is defined and the function $\gamma: \operatorname{dom}(\gamma) \rightarrow N_{1}$. Also, $t_{0} \in(a, b)$. Moreover, above $\gamma((a, b))=\sigma_{N_{1}}(z, \xi)$ means that the sets $\gamma((a, b)) \subset N_{1}$ and $\sigma_{N_{1}}(z, \xi) \subset N_{1}$ are the same, or equivalently, that $\gamma((a, b))$ and $\sigma_{N_{1}}(z, \xi)$ are the same as unparameterized curves.

For a moment we consider only metric $g_{1}$. Assume that $p$ is a point in $N_{1}$ and $q$ is point of $F_{1}^{\text {int }}$ such that $q=\gamma_{p, \xi}(\ell), \ell>0$ and the geodesic $\gamma_{p, \xi}([0, \ell])$ has no cut points. Then there is a neighborhood $U \subset F_{1}^{\text {int }}$ of $q$ and a neighborhood $V \subset T_{p} N_{1}$ of $\ell \xi$ such that $\exp _{p}: V \rightarrow U$ is a diffeomorphism. Assuming that the neighborhood $V$ is small enough, we see that for any $\ell v \in V,\|v\|_{g_{1}}=1$, there is $s>0$ such that the geodesic $\gamma_{p, v}([-s, \ell])$ has no cut points. Let $s_{0}(p, v) \in(0, \infty]$ be the supremum of such $s$. Then, for the geodesic $\gamma_{p, v}:\left(-s_{0}(p, v), \ell\right) \rightarrow N_{1}$ we have $\left(\gamma_{p, v}, 0\right) \in \mathcal{C}(p)$. This proves that set

$$
\begin{aligned}
\Omega_{p}:=\left\{v \in T_{p} N_{1} \quad ;\right. & \text { there are }\left(c, t_{p}\right) \in \mathcal{C}(p), c\left(t_{p}\right)=p \\
& \text { and } \left.\dot{c}\left(t_{p}\right) \text { is proportional to } \pm v\right\}
\end{aligned}
$$

contains a non-empty open double cone $\Sigma_{p}$, that is, an open set that satisfies $r v \in \Sigma_{p}$ for all $v \in \Sigma_{p}$ and $r \in \mathbb{R} \backslash\{0\}$. Note that the complement of $\Omega_{p}$ in $T_{p} N_{1}$ is non-empty, if in manifold $M_{1}$ there are closed geodesics, or geodesics that are trapping in both directions in $M_{1}$ and go through the point $p$.

Let point $p \in N_{1}$ and $(U, X)$ be coordinates near $p, X: U \rightarrow \mathbb{R}^{n}$, and denote $X(q)=\left(x^{j}(q)\right)_{j=1}^{n}$. Recall that a pre-geodesic $\widetilde{\gamma}$ on $\left(N_{1}, g_{1}\right)$ satisfies the formula (11), that is,

$$
\left.\left[\frac{d^{2} \widetilde{\gamma}^{k}}{d s^{2}}(s)+\Gamma_{i, j}^{k}(\widetilde{\gamma}(s)) \frac{d \widetilde{\gamma}^{i}}{d s}(s) \frac{d \widetilde{\gamma}^{j}}{d s}(s)\right]\right|_{s=s_{p}}=\left.f\left(\frac{d \widetilde{\gamma}}{d s}\right) \frac{d \widetilde{\gamma}^{k}}{d s}(s)\right|_{s=s_{p}},
$$

$k \in\{1,2, \ldots, n\}$. Here $\gamma\left(s_{p}\right)=p$ and $f$ is some function that is homogeneous of degree 1 on the subbundle of $T U$ that is determined by the velocity vectorfield of $\widetilde{\gamma}$.

Next, we change the point of view and consider the equation (28) as a system of equations for the "unknown" $(\Gamma, f)$ with the given coefficients 


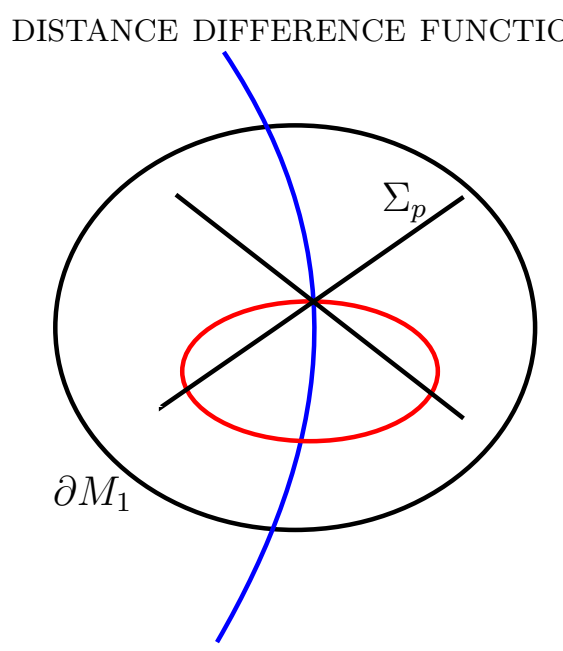

FiguRE 4. For all $p \in M_{1}$ there exists an open conic set $\Sigma_{p} \subset T_{p} N_{1}$ such that for every $\xi \in \Sigma_{p}$ the geodesic $\gamma_{p, \xi}$ of $\left(N_{1}, g_{1}\right)$ can be extended to a distance minimizing geodesic (blue curve in the figure) that enters the set $F=$ $N \backslash M$. When the distance difference data for $g_{1}$ and $g_{2}$ coincide, these geodesics have to be pre-geodesic also with respect to the metric $\Psi^{*} g_{2}$. Note that there may be $g_{1}$ geodesics emanating from $p$ to directions $\xi \notin \Sigma_{p}$ that do not intersect the set $F$. Such geodesics can be e.g. closed loops in $M_{1}$ (red curve).

$\left.\frac{d \widetilde{\gamma}}{d s}(s)\right|_{s=s_{p}} \in \Omega_{p}$ and $\left.\frac{d^{2} \widetilde{\gamma}}{d s^{2}}(s)\right|_{s=s_{p}}$ where $\left(\widetilde{\gamma}, s_{p}\right) \in \mathcal{C}(p)$. Here $\Gamma$ stands for a collection of Christoffel symbols $\Gamma_{i, j}^{k}$ and $f: \Omega \rightarrow \mathbb{R}$ is a function that satisfies equation (10) on the subbundle

$$
\Omega:=\bigcup_{p \in U} \Omega_{p} \subset T U
$$

Suppose that we also have another Riemannian connection which Christoffel symbols $\widetilde{\Gamma}_{i, j}^{k}$ in the $(U, X)$-coordinates have the form

$$
\widetilde{\Gamma}_{i, j}^{k}=\Gamma_{i, j}^{k}+\delta_{i}^{k} \varphi_{j}+\delta_{j}^{k} \varphi_{i}
$$

for some smooth functions $\varphi_{i}: U \rightarrow \mathbb{R}, i=1,2, \ldots, n$. Here, $\delta_{i}^{k}$ is one when $k=i$ and zero otherwise. Let $\varphi(x)=\varphi_{i}(x) d x^{i}$ be a smooth 1-form that has functions $\left(\varphi_{i}\right)_{i=1}^{n}$ as the coefficients. We need the following consequence of Lemma 2.1:

Lemma 2.11. Let $(U, X)$ a smooth coordinate chart. If the Christoffel symbols $\widetilde{\Gamma}$ and $\Gamma$ satisfy the equation (29) for some 1-form $\varphi$ and pair $(f, \Gamma), f: \Omega \rightarrow \mathbb{R}$ is homogenous of degree 1 , is a solution of (11), with $s=s_{p}$, for all $\left(\widetilde{\gamma}, s_{p}\right) \in \mathcal{C}(p)$, then pair $(\widetilde{\Gamma}, \widetilde{f})$ where

$$
\tilde{f}(v)=f(v)+2 \varphi(v), v \in \Omega .
$$

is also a solution of $(11)$, with $s=s_{p}$, for all $\left(\widetilde{\gamma}, s_{p}\right) \in \mathcal{C}(p)$. 
Proof. Let $\left(\widetilde{\gamma}, s_{p}\right) \in \mathcal{C}(p)$. A direct computation shows that

$$
\begin{gathered}
\left(\delta_{i}^{k} \varphi_{j}+\delta_{j}^{k} \varphi_{i}\right) \frac{d \widetilde{\gamma}^{i}}{d s}(s) \frac{d \widetilde{\gamma}^{j}}{d s}(s)=\varphi_{j} \frac{d \widetilde{\gamma}^{k}}{d s}(s) \frac{d \widetilde{\gamma}^{j}}{d s}(s)+\varphi_{i} \frac{d \widetilde{\gamma}^{i}}{d s}(s) \frac{d \widetilde{\gamma}^{k}}{d s}(s) \\
=2 \frac{d \widetilde{\gamma}^{k}}{d s}(s)\left(\varphi_{i} \frac{d \widetilde{\gamma}^{i}}{d s}(s)\right)=2 \frac{d \widetilde{\gamma}^{k}}{d s}(s) \varphi\left(\frac{d \widetilde{\gamma}}{d s}(s)\right)
\end{gathered}
$$

Use this and substitute equation (29) into equation (11) to obtain

$$
\frac{d^{2} \widetilde{\gamma}^{k}}{d s^{2}}(s)+\left.\widetilde{\Gamma}_{i, j}^{k}(p) \frac{d \widetilde{\gamma}^{i}}{d s}(s) \frac{d \widetilde{\gamma}^{j}}{d s}(s)\right|_{s=s_{p}}=\frac{d \widetilde{\gamma}^{k}}{d s}(s)\left[f\left(\frac{d \widetilde{\gamma}}{d s}(s)\right)+2 \varphi\left(\frac{d \widetilde{\gamma}}{d s}(s)\right)\right]
$$

that proves the claim.

The following lemma gives a converse result for Lemma 2.11. It is obtained by using, in a quite straightforward way, results of V. Matveev [46, Sec. 2] for general affine connections on pseudo-Riemannian manifolds. However, for the convenience of the reader, we give a detailed proof for the lemma and analyze at the same time the smoothness of the 1-form $x \mapsto \varphi(x)$ in a local coordinate neighborhood $U \subset M$.

Lemma 2.12. Let $(U, X)$ a smooth coordinate chart. Let functions $f: \Omega \rightarrow \mathbb{R}$ and $\tilde{f}: \Omega \rightarrow \mathbb{R}$ be homogeneous of degree 1. Suppose that pairs $(f, \Gamma)$ and $(\widetilde{\Gamma}, \widetilde{f})$ both solve at all points $p \in U$ the system (11) for all such coefficients $\left.\frac{d \gamma}{d s}(s)\right|_{s=s_{p}} \in \Omega_{p}$ and $\left.\frac{d^{2} \gamma}{d s^{2}}(s)\right|_{s=s_{p}}$ that $\left(\gamma, s_{p}\right) \in \mathcal{C}(p)$. Then the Christoffel symbols $\Gamma$ and $\widetilde{\Gamma}$ satisfy equation (29) in $U$ with a $C^{\infty}$-smooth 1-form $\varphi$ in $U$.

Proof. Define a pair $(\bar{f}, \bar{\Gamma})$ as

$$
\bar{f}=f-\tilde{f} \text { and } \bar{\Gamma}_{i, j}^{k}=\Gamma_{i, j}^{k}-\widetilde{\Gamma}_{i, j}^{k}
$$

As a difference of two connection coefficients, $\bar{\Gamma}$ is a tensor. By substitution of pairs $(f, \Gamma)$ and $(\widetilde{\Gamma}, \widetilde{f})$ into equation (11) and by subtracting the obtained equations, we obtain at $p \in U$

$$
\bar{\Gamma}_{i, j}^{k} v^{i} v^{j}=\bar{f}(v) v^{k}, \text { for every } v \in \Omega_{p} .
$$

Note that (32) defines a smooth extension of $\bar{f}: \Omega \rightarrow \mathbb{R}$ to $T U \backslash\{0\}$, given by

$$
\bar{f}(v)=\frac{\bar{f}(v) v^{k} g_{k \ell} v^{\ell}}{g(v, v)}=\frac{\bar{\Gamma}_{i, j}^{k}(p) v^{i} v^{j} g_{k \ell}(p) v^{\ell}}{g_{a b}(p) v^{a} v^{b}},(p, v) \in T U \backslash\{0\} .
$$

Here, the rightmost term is smooth in $T U \backslash\{0\}$.

Recall that $\Omega_{p}$ contains an open double cone $\Sigma_{p} \subset \Omega_{p}$. Our next goal is to show that there exist a linear function $\varphi: T_{p} N \rightarrow \mathbb{R}$ such that the restriction of function $\bar{f}$, to $\Sigma_{p} \subset \Omega_{p}$, is equal to $\left.2 \varphi\right|_{\Sigma_{p}}$. Define a family of symmetric bi-linear mappings

$$
\sigma^{k}: T_{p} N \times T_{p} N \rightarrow \mathbb{R}, \sigma^{k}(u, v)=\bar{\Gamma}_{i, j}^{k} v^{i} u^{j}, k \in\{1, \ldots, n\} .
$$


Since mappings $\sigma^{k}$ are symmetric, the parallelogram equation

$$
0=\sigma^{k}(u+v, u+v)+\sigma^{k}(u-v, u-v)-2 \sigma^{k}(u, u)-2 \sigma^{k}(v, v)
$$

holds.

Next, let $u \in \Sigma_{p}, u \neq 0$. Then there is $\varepsilon=\varepsilon(u)>0$ such that, if $v \in T_{p} N$ satisfies $\|v\|<\varepsilon$, then $u-v \in \Sigma_{p}$.

Let us next consider $v \in \Sigma_{p}$ with $\|v\|<\varepsilon$. Then $u-v, u+v \in \Sigma_{p} \subset$ $\Omega_{p}$. By the parallelogram equality for the function $\sigma^{k}$ and (32) we have (34)

$$
\begin{aligned}
0 & =\bar{f}(u+v)(u+v)+\bar{f}(u-v)(u-v)-2 \bar{f}(u) u-2 \bar{f}(v) v \\
& =(\bar{f}(u+v)+\bar{f}(u-v)-2 \bar{f}(u)) u+(\bar{f}(u+v)-\bar{f}(u-v)-2 \bar{f}(v)) v .
\end{aligned}
$$

If vectors $u$ and $v$ are linearly independent, we get a system

$$
\left\{\begin{array}{l}
\bar{f}(u+v)+\bar{f}(u-v)-2 \bar{f}(u)=0 \\
\bar{f}(u+v)-\bar{f}(u-v)-2 \bar{f}(v)=0 .
\end{array}\right.
$$

By summing up these two equations, we get

$$
\bar{f}(u+v)=\bar{f}(u)+\bar{f}(v) .
$$

Observe that the system (35) is valid also when $v=\lambda u, \lambda \in \mathbb{R}$. Recall that the mappings $f$ and $\widetilde{f}$ are solutions of (11) and therefore, they satisfy the equation (10), i.e., they commute with scalar multiplication in $\Omega_{p}$.

So far we have proved that $\bar{f}(u+\cdot)$ and $\bar{f}(u)+\bar{f}(\cdot)$ coincide in set $B_{p}(0, \epsilon) \cap \Sigma_{p}$. Since $\bar{f}$ is homogeneous of degree 1 it holds by (36) that

$$
\bar{f}(u+a v)=\bar{f}(u)+a \bar{f}(v), v \in B_{p}(0, \epsilon) \cap \Sigma_{p},-1<a<1 .
$$

We define a linear function

$$
2 \varphi: T_{p} N \rightarrow \mathbb{R}, 2 \varphi(v)=\lim _{r \rightarrow 0} \frac{\bar{f}(u+r v)-\bar{f}(u)}{r}=\nabla_{u} \bar{f}(u) \cdot v .
$$

If $v \in \Sigma_{p}$ and $r$ is small enough, then $r v \in B_{p}(0, \varepsilon) \cap \Sigma_{p}$ and therefore by formula (37) it holds that

$$
2 \varphi(v)=\bar{f}(v) \quad \text { for every } v \in \Sigma_{p} .
$$

As $\Sigma_{p}$ is open, and $\varphi$ and $\bar{f}$ are linear, this holds for all $v \in T_{p} N$ and thus $\varphi(v)$ given by the formula (38) is independent on the choice of used $u \in \Sigma_{p}$. In local coordinates $(U, X)$ we have by (33) and (39) that

$$
\varphi\left(\frac{\partial}{\partial x^{\ell}}\right):=\frac{1}{2} \sum_{i, k, j=1}^{n} \frac{1}{g_{\ell \ell}(x)} \bar{\Gamma}_{i, j}^{k}(x) \delta_{\ell}^{i} \delta_{\ell}^{j} g_{k \ell}(x)
$$

defines a $C^{\infty}$-smooth 1 -form $x \mapsto \varphi(x)$ in $U$, that is an extension of $\bar{f}: \Omega \rightarrow \mathbb{R}$.

Define a connection

$$
\widehat{\Gamma}_{i, j}^{k}:=\widetilde{\Gamma}_{i, j}^{k}+\delta_{i}^{k} \varphi_{j}+\delta_{j}^{k} \varphi_{i},
$$


and choose $v=\left.\frac{d}{d s} \gamma(s)\right|_{s=s_{p}} \in \Sigma_{p}$. Since pairs $(f, \Gamma)$ and $(\widetilde{\Gamma}, \widetilde{f})$ are both solutions of (11) the above considerations yield

$$
\begin{aligned}
& {\left.\left[\frac{d^{2} \gamma^{k}}{d s^{2}}(s)+\Gamma_{i, j}^{k}(p) \frac{d \gamma^{i}}{d s}(s) \frac{d \gamma^{j}}{d s}(s)\right]\right|_{s=s_{p}}=\left.\left[f\left(\frac{d \gamma}{d s}(s)\right) \frac{d \gamma^{k}}{d s}(s)\right]\right|_{s=s_{p}} } \\
= & \left.\frac{d \gamma^{k}}{d s}(s)\left[2 \varphi\left(\frac{d \gamma}{d s}(s)\right)+\widetilde{f}\left(\frac{d \gamma}{d s}(s)\right)\right]\right|_{s=s_{p}} \\
= & {\left.\left[\frac{d^{2} \gamma^{k}}{d s^{2}}(s)+\widetilde{\Gamma}_{i, j}^{k}(p) \frac{d \gamma^{i}}{d s}(s) \frac{d \gamma^{j}}{d s}(s)\right]\right|_{s=s_{p}}+\left.\frac{d \gamma^{k}}{d s}(s)\left[2 \varphi\left(\frac{d \gamma}{d s}(s)\right)\right]\right|_{s=s_{p}} } \\
\stackrel{(31)}{=} & {\left.\left[\frac{d^{2} \gamma^{k}}{d s^{2}}(s)+\widehat{\Gamma}_{i, j}^{k}(p) \frac{d \gamma^{i}}{d s}(s) \frac{d \gamma^{j}}{d s}(s)\right]\right|_{s=s_{p}} . }
\end{aligned}
$$

Therefore we have

$$
\left.\Gamma_{i, j}^{k}(p) \frac{d \gamma^{i}}{d s}(s) \frac{d \gamma^{j}}{d s}(s)\right|_{s=s_{p}}=\left.\widehat{\Gamma}_{i, j}^{k}(p) \frac{d \gamma^{i}}{d s}(s) \frac{d \gamma^{j}}{d s}(s)\right|_{s=s_{p}} .
$$

Thus we have shown that for all $v \in \Sigma_{p}$ the equation

$$
\Gamma_{i, j}^{k}(p) v^{i} v^{j}=\widehat{\Gamma}_{i, j}^{k}(p) v^{i} v^{j}
$$

is valid. Since set $\Sigma_{p}$ is open, it holds that

$$
\Gamma_{\ell, m}^{k}(p)=\partial_{v^{\ell} v^{m}} \Gamma_{i, j}^{k}(p) v^{i} v^{j}=\partial_{v^{\ell} v^{m}} \widehat{\Gamma}_{i, j}^{k}(p) v^{i} v^{j}=\widehat{\Gamma}_{\ell, m}^{k}(p) .
$$

As above $p \in U$ is arbitrary, this proves the claim.

Proposition 2.13. Suppose that Riemannian manifolds $\left(N_{1}, g_{1}\right)$ and $\left(N_{2}, g_{2}\right)$ are as in Section 1.2 and (2)-(3) are valid. Let $p \in N_{1}$ and $(U, X)$ be coordinates in a neighborhood of $p$. Then it holds that the Christoffel symbols $\Gamma$ and $\widetilde{\Gamma}$ of metrics $g_{1}$ and $\left(\Psi^{-1}\right)^{*} g_{2}$, respectively, satisfy equation (29) in $U$ with some 1-form $\varphi$, where $\Psi$ is as in (20).

Proof. Let $(U, X)$ be a local coordinate system in $N_{1}$. Our aim is to use the Lemma 2.12 to prove the claim of this Lemma. To do so we need to construct a function $\tilde{f}: \Omega \rightarrow \mathbb{R}$ that satisfies (10) and moreover for any $q \in U$ the pair $(\widetilde{\Gamma}, \widetilde{f})$ solves the system (11) for all such coefficients $\left.\frac{d \gamma}{d s}(s)\right|_{s=s_{q}} \in \Omega_{q}$ and $\left.\frac{d^{2} \gamma}{d s^{2}}(s)\right|_{s=s_{q}}$ that $\left(\gamma, s_{q}\right) \in \mathcal{C}(q)$.

Let $p \in U$ and $\left(c_{1}, t_{1}\right) \in \mathcal{C}(p)$. With out loss of generality we may assume that $t_{1}=0$ and $\dot{c}_{1}(0)=\xi \in S_{p} N \cap \Omega_{p}$. By definition of $\mathcal{C}(p)$, it holds that there is a unique reparametrization $t \mapsto s_{\xi}(t)=: s(t)$ of $c_{1}$ such that for curves $c_{1}$ and $c_{2}=c_{1} \circ s$ we have $s(0)=p, \dot{c}_{2}(0)=\xi$ and

$$
\left\{\begin{array}{l}
\ddot{c}_{1}^{k}(t)+\dot{c}_{1}^{i}(t) \dot{c}_{1}^{j}(t) \Gamma_{i, j}^{k}\left(c_{1}(t)\right)=0, \\
\ddot{c}_{2}^{k}(t)+\dot{c}_{2}^{i}(t) \dot{c}_{2}^{j}(t) \widetilde{\Gamma}_{i, j}^{k}\left(c_{2}(t)\right)=0 .
\end{array}\right.
$$

Using the chain rule we can write the latter equation as

$$
\ddot{c}_{1}^{k}(s(t))+\dot{c}_{1}^{i}(s(t)) \dot{c}_{1}^{j}(s(t)) \widetilde{\Gamma}_{i, j}^{k}\left(c_{1}(s(t))\right)=-\frac{\ddot{s}(t)}{\dot{s}(t)^{2}} \dot{c}_{1}^{k}(s(t)) .
$$


We define $f: \Omega \rightarrow \mathbb{R}$

$$
f(q, v)=\frac{\ddot{s}_{v}(t)}{\dot{s}_{v}(t)^{2}}, \text { if } v=\dot{\gamma}(0) \text { for some }(\gamma, 0) \in \mathcal{C}(q) .
$$

Above $s_{v}$ is such a reparametrization of $\gamma$ that, $s_{v}(0)=0$,

$\left.\frac{d}{d t} \gamma(s(t))\right|_{t=0}=v$ and (42) is valid, when $c_{1}$ is replaced with $\gamma$ and $c_{2}$ is replaced with $\gamma \circ s_{v}$. Note that function $f$ is well defined and satisfies the equation (10), since geodesic equation (7) is preserved under affine re-parametrizations. Therefore it holds that for any $q \in U$ the pairs $(\Gamma, 0)$ and $(\widetilde{\Gamma}, f)$ both solve the system $(11)$ for all such coefficients $\left.\frac{d \gamma}{d s}(s)\right|_{s=s_{q}} \in \Omega_{q}$ and $\left.\frac{d^{2} \gamma}{d s^{2}}(s)\right|_{s=s_{q}}$ that $\left(\gamma, s_{q}\right) \in \mathcal{C}(q)$. The claim follows then from Lemma 2.12 .

Lemma 2.14. Suppose that the connections $\Gamma$ and $\widetilde{\Gamma}$ corresponding to metric tensors $g$ and $\widetilde{g}$, respectively, satisfy the equation (29) with a 1 -form $\varphi$. Then the metric tensors $g$ and $\widetilde{g}$ are geodesically equivalent.

Proof. Let $\gamma(t)$ be a geodesic with respect to the metric $g$. Then $\gamma$ satisfies the geodesic equation (7). Substitute $\Gamma$ with $\widetilde{\Gamma}$ into (7) to get the equation

$$
\frac{d^{2} \gamma^{k}}{d t^{2}}(t)+\widetilde{\Gamma}_{i, j}^{k}(\gamma(t)) \frac{d \gamma^{i}}{d t}(t) \frac{d \gamma^{j}}{d t}(t)=2 \frac{d \gamma^{k}}{d t}(t) \varphi\left(\frac{d \gamma}{d t}(t)\right)
$$

Write $\kappa(t)=2 \varphi(\dot{\gamma}(t))$ and use Lemma 2.1 to show that there exists a change of parameters $s \mapsto t(s)$ such that $s \mapsto \gamma(t(s))$ is a geodesic with respect to the metric $\widetilde{\Gamma}$. As the roles of $g$ and $\widetilde{g}$ can be exchanged, the claim follows.

By the Lemma 2.14, the equivalence of the distance difference data (2)-(3) implies the geodesic equivalence of metric tensors $g$ and $\Psi_{*} g_{2}$ on $N_{1}$. In the following theorem, that shows that metric tensors $g$ and $\Psi_{*} g_{2}$ coincide also in $N_{1}$, we will use the implications of the MatveevTopalov theorem [43]. Their result is also concerned in the appendix of the extended preprint version of this paper [37] and its generalizations have been considered in [11, 63].

Lemma 2.15. Suppose that manifold $N$ satisfies assumptions of Section 1.2 and $g$ and $\widetilde{g}$ are two metric tensors on $N$. Suppose that these metrics are geodesically equivalent on manifold $N$ and coincide in set $F^{\text {int }} \neq \emptyset$. Then $g=\widetilde{g}$ in whole $N$.

Proof. Define a smooth mapping $I_{0}: T N \rightarrow \mathbb{R}$ as

$$
I_{0}((x, v))=\left(\frac{\operatorname{det}\left(g_{x}\right)}{\operatorname{det}\left(\widetilde{g}_{x}\right)}\right)^{\frac{2}{n+1}} \widetilde{g}_{x}(v, v),
$$

where $\widetilde{g}_{x}(v, v)=\widetilde{g}_{j k}(x) v^{j} v^{k}$. Note that the function $x \mapsto \frac{\operatorname{det}\left(g_{x}\right)}{\operatorname{det}\left(\widetilde{g}_{x}\right)}$ is coordinate invariant. 
Let $\gamma_{g}$ be a geodesic of metric $g$. Define a smooth path $\beta$ in $T N$ as $\beta(t)=\left(\gamma_{g}(t), \dot{\gamma}_{g}(t)\right)$, i.e., $\beta$ is an integral curve of the geodesic flow of metric $g$. The Matveev-Topalov theorem [43] states that if $g$ and $\widetilde{g}$ are geodesically equivalent, then there are several invariants related to the $(1,1)$-tensor $G=g^{-1} \widetilde{g}$, given in local coordinates by $G_{k}^{j}(x)=$ $g^{j i}(x) \widetilde{g}_{i k}(x)$, that are constants along integral curves $\beta(t)$. In particular, the function $t \mapsto I_{0}(\beta(t))$ is a constant.

A corollary of Matveev-Topalov theorem, [43, Cor. 2] (see also [44, Cor. 2] and [11, Thm. 3]), is that the number $n(x)$ of the different eigenvalues of the map $G(x): T_{x} N \rightarrow T_{x} N$ is constant at almost every point $x \in N$. Since $G(x)=I$ for $x \in F^{\text {int }}$, we have that $n(x)=0$ in the set $F^{\text {int }}$ having a positive measure. This implies that $n(x)=0$ for almost all $x \in N$. Hence for almost all $x \in N$ there is $c(x) \in \mathbb{R}_{+}$ such that we have $G(x)=c(x) I$, so that $\widetilde{g}_{i k}(x)=c(x) g_{i k}(x)$. As $G$ is continuous, this holds for all $x \in N$. Summarizing, the first implication of the Matveev-Topalov theorem is that $g$ and $\widetilde{g}$ are conformal on the whole manifold $N$.

Let $x_{0}$ be a point of $N$. Since we assumed that metrics $g$ and $\widetilde{g}$ coincide in set $F$, we have for any point $z \in F$ and vector $v \in T_{z} N$ that formula (43) has form

$$
I_{0}(z, v)=\widetilde{g}_{z}(v, v)=g_{z}(v, v) .
$$

Let $\gamma(t):=\gamma_{z, \xi}^{g}(t), \xi \in S_{z} N, z \in F$ be a $g$-geodesic passing through $x_{0}$ such that $x_{0}=\gamma\left(t_{0}\right)$ for some $t_{0} \geq 0$. The $I_{0}((z, \xi))=1$ and by the Matveev-Topalov theorem, $I_{0}$ is constant along the integral curves of geodesic flow of $g$. Thus, we have

$$
I_{0}\left(x_{0}, \dot{\gamma}\left(t_{0}\right)\right)=I_{0}(z, \xi)=1 .
$$

Define $W_{x_{0}}$ to be the set of all $g$-unit vectors of $T_{x_{0}} N$ with respect to metric $g$, such that every vector in $W_{x_{0}}$ is a velocity vector of some $g$-geodesic starting from $F$ and passing trough $x_{0}$. Recall that set $W_{x_{0}}^{\text {int }} \subset S_{x_{0}} N$ is not empty.

Let $X=\left(x^{1}, \ldots, x^{n}\right)$ be any coordinate chart at $x_{0}$. Formula (45) shows that for every $\xi \in W_{x_{0}}$ we have

$$
g_{i j}\left(x_{0}\right) \xi^{i} \xi^{j}=1=I_{0}\left(x_{0}, \xi\right)=\left(\frac{\operatorname{det}\left(g_{x_{0}}\right)}{\operatorname{det}\left(\widetilde{g}_{x_{0}}\right)}\right)^{\frac{2}{n+1}} \widetilde{g}_{i j}\left(x_{0}\right) \xi^{i} \xi^{j}
$$

Consider an open cone

$$
W_{x_{0}}^{\text {int }} \cdot \mathbb{R}_{+}:=\left\{t w \in T_{x_{0}} N: t>0, w \in W_{x_{0}}^{\text {int }}\right\} .
$$

Then the equation (46) holds for all $\xi \in W_{x_{0}}^{\text {int }} \cdot \mathbb{R}_{+}$and since the set $W_{x_{0}}^{\text {int }} \cdot \mathbb{R}_{+}$is open and both sides of equation (46) are smooth in $\xi$, we obtain the equation

$$
g_{i j}\left(x_{0}\right)=\left(\frac{\operatorname{det}\left(g_{x_{0}}\right)}{\operatorname{det}\left(\widetilde{g}_{x_{0}}\right)}\right)^{\frac{2}{n+1}} \widetilde{g}_{i j}\left(x_{0}\right), \text { for all } i, j \in\{1, \ldots, n\},
$$


as a second order derivative with respect to $\xi$ of equation (46).

Denote $f(p):=\frac{\operatorname{det}(g(p))}{\operatorname{det}(\widetilde{g}(p))}$. Then the above yields

$$
\left(f\left(x_{0}\right)\right)^{\frac{2}{n+1}} \widetilde{g}_{j k}\left(x_{0}\right)=g_{j k}\left(x_{0}\right), \text { for all } j, k \in\{1, \ldots, n\} .
$$

Taking determinants of both sides of (48) we see that

$$
\left(f\left(x_{0}\right)\right)^{\frac{2 n}{n+1}-1}=1 .
$$

Since we we have assumed the dimension of manifold $N$ is at least 2, we see from equation (49) that $f\left(x_{0}\right)=1$. By formula (48) this implies $g=\tilde{g}$ on $M$.

Theorem 1.3 follows now from Theorems 2.4 and 2.7 and Lemmas 2.14 and 2.15 .

\section{Application For An inVERSE PROBlem For A WAVE EQUATION}

Here we consider an application of Theorem 1.3 for an inverse problem for a wave equation with spontaneous point sources.

3.0.1. Support sets of waves produced by point sources. Let $(N, g)$ be a closed Riemannian manifold. Denote the Laplace-Beltrami operator of metric $g$ by $\Delta_{g}$. We consider a wave equation

$$
\left\{\begin{array}{l}
\left(\partial_{t}^{2}-\Delta_{g}\right) G(\cdot, \cdot, y, s)=\kappa(y, s) \delta_{y, s}(\cdot, \cdot), \quad \text { in } \mathcal{N} \\
G(x, t, y, s)=0, \quad \text { for } t<s, x \in N
\end{array}\right.
$$

where $\mathcal{N}=N \times \mathbb{R}$ is the space-time. The solution $G(x, t, y, s)$ is the wave produced by a point source located at the point $y \in M$ and time $s \in \mathbb{R}$ having the magnitude $\kappa(y, s) \in \mathbb{R} \backslash\{0\}$. Above, we have $\delta_{y, s}(x, t)=\delta_{y}(x) \delta_{s}(t)$ corresponds to a point source at $(y, s) \in \mathcal{N}$.

3.0.2. Inverse coefficient problem with spontaneous point source data. Assume that there are two manifolds $\left(N_{1}, g_{1}\right)$ and $\left(N_{2}, g_{2}\right)$ satisfying the assumptions given in Section 1.2 and

$$
\text { There exists an isometry } \phi: F_{1} \rightarrow F_{2}
$$$$
W_{1}=W_{2}
$$

where $W_{1}$ and $W_{2}$ are collections of supports of waves produced by point sources taking place at unknown points at unknown time, that is,

$$
W_{1}=\left\{\operatorname{supp}\left(G^{1}\left(\cdot, \cdot, y_{1}, s_{1}\right)\right) \cap\left(F_{1} \times \mathbb{R}\right) ; y_{1} \in M_{1}, s_{1} \in \mathbb{R}\right\} \subset 2^{F_{1} \times \mathbb{R}}
$$

and

$W_{2}=\left\{\operatorname{supp}\left(G^{2}\left(\phi(\cdot), \cdot, y_{2}, s_{2}\right)\right) \cap\left(F_{1} \times \mathbb{R}\right) ; y_{2} \in M_{2}, s_{2} \in \mathbb{R}\right\} \subset 2^{F_{1} \times \mathbb{R}}$ where functions $G^{j}, j=\{1,2\}$ solve equation (50) on manifold $N_{j}$. Here $2^{F_{j} \times \mathbb{R}}=\left\{V ; V \subset F_{j} \times \mathbb{R}\right\}$ is the power set of $F_{j} \times \mathbb{R}$. Roughly speaking, $W_{j}$ corresponds to the data that one makes by observing, in 
the set $F_{j}$, the waves that are produced by spontaneous point sources that that go off, at an unknown time and at an unknown location, in the set $M_{j}$.

Earlier, the inverse problem for the sources that are delta-distributions in time and localized also in the space has been studied in [16] in the case when the metric $g$ is known. Theorem 1.3 yields the following result telling that the metric $g$ can be determined when a large number of waves produced by the point sources are observed:

Proposition 3.1. Let $\left(N_{j}, g_{j}\right), j=1,2$ be a closed compact Riemannian $n$-manifolds, $n \geq 2$ and $M_{j} \subset N_{j}$ be an open set such that $F_{j}=N_{j} \backslash M_{j}$ have non-empty interior. If the spontaneous point source data of these manifolds coincide, that is, we have (51)-(52), then $\left(N_{1}, g_{1}\right)$ and $\left(N_{2}, g_{2}\right)$ are isometric.

Proof. Let us again omit the sub-indexes of $N, M$, and $F$. For $y \in M$, $s \in \mathbb{R}$, and $z \in F$ we define a number

$$
\begin{gathered}
\mathcal{T}_{y, s}(z)=\sup \{t \in \mathbb{R} ; \text { the point }(z, t) \text { has a neighborhood } \\
\left.U \subset \mathcal{N} \text { such that }\left.G(\cdot, \cdot, y, s)\right|_{U}=0\right\}
\end{gathered}
$$

which tells us, what is the first time when the wave $G(\cdot, \cdot, y, s)$ is observed near the point $z$. Using the finite velocity of the wave propagation for the wave equation, see [21], we see that the support of $G(\cdot, \cdot, y, s)$ is contained in the future light cone of the point $q=(y, s) \in$ $\mathcal{N}$ given by

$$
J^{+}(q)=\left\{\left(y^{\prime}, s^{\prime}\right) \in \mathcal{N} ; s^{\prime} \geq d\left(y^{\prime}, y\right)+s\right\} .
$$

Next, for $\xi=\xi^{j} \frac{\partial}{\partial x^{j}} \in T_{y} N$ we denote the corresponding co-vector by $\xi^{b}=g_{j k}(y) \xi^{j} d x^{j}$. Then the results of [18] and [19] on the propagation of singularities for the real principal type operators, in particular for the wave operator, imply that in the set $\mathcal{N} \backslash\{q\}$ Green's function $G(\cdot, \cdot, y, s)$ is a Lagrangian distribution associated to the Lagranian sub-manifold

$$
\Sigma_{0}=\left\{\left(\gamma_{y, \eta}(t), s+t ; \dot{\gamma}_{y, \eta}(t)^{b}, d t\right) \in T^{*} \mathcal{N} ; \eta \in S_{y} N, t>0\right\}
$$

and its principal symbol on $\Sigma_{0}$ is non-zero. In particular, [19, Prop. 2.1] implies that $\Sigma=\Sigma_{0} \cup\left(T_{q}^{*} \mathcal{N}, \backslash\{0\}\right)$ coincides with the wave front set $\operatorname{WF}(u)$ of the solution $u=G(\cdot, \cdot, y, s)$. This means that a wave emanating from a point source $(y, s)$ propagates along the geodesics of manifold $(N, g)$. The image of $\operatorname{WF}(u)$ in the projection $\pi: T^{*} \mathcal{N} \rightarrow \mathcal{N}$ coincides the singular support of $u$. Hence, we see that

$$
\begin{aligned}
& \operatorname{singsupp}(G(\cdot, \cdot, y, s))=S(q), \quad \text { where } \\
& S(q)=\left\{\left(\exp _{y}(t \eta), s+t\right) \in \mathcal{N} ; \eta \in S_{y} N, t \geq 0\right\} .
\end{aligned}
$$

Since the Riemannian manifold $N$ is complete, the space-time $\mathcal{N}$ is a globally hyperbolic Lorentzian manifold and we have $\partial J^{+}(q)=S(q)$, see [50]. Summarizing, the above implies that the function $G(\cdot, \cdot, y, s)$ vanishes outside $J^{+}(q)$ and is non-smooth, and thus it is non-vanishing 
in a neighborhood of arbitrary point of $\partial J^{+}(q)$. Thus, for $z \in F$ we have $\mathcal{T}_{y, s}(z)=d(z, y)-s$. Hence the distance difference functions satisfy equation

$$
D_{y}\left(z_{1}, z_{2}\right)=\mathcal{T}_{y, s}\left(z_{1}\right)-\mathcal{T}_{y, s}\left(z_{2}\right) .
$$

Thus, when formulas (51)-(52) are valid, we see using equation (54) that the distance difference data of the manifolds $N_{1}$ and $N_{2}$ coincide, that is, we have (2)-(3). Hence, the claim follows from Theorem 1.3.

Finally, we note that sets $W_{j}$ are closely related to the light-observation sets studied in [34] in the study of the inverse problems for non-linear hyperbolic problems with a time-dependent metric. The light-observation set $P_{U}(q)$ corresponding to a source point $q=(y, s)$ and the observation set $U$ is the intersection of $U$ and the future light cone emanating from $q$. In fact, the formula (53) implies that in the space time $\mathcal{N}=N \times \mathbb{R}$ the sets $W_{j}$ coincide with the light-observation sets $P_{U}(q)$ corresponding to a source point $q=(y, s)$ and the observation set $U=F \times \mathbb{R}$.

Acknowledgements. The authors express their gratitude to Institut Henri Poincare, where part of this work was done. The authors thank prof. V. Matveev and P. Topalov for valuable comments and prof. M. de Hoop for pointing out the relation of geometric inverse problems and the geophysical measurements of microearthquakes.

ML is partially supported by the Finnish Centre of Excellence in Inverse Problems Research 2012-2017 and an Academy Professorship. TS is partially supported by Academy of Finland, project 263235.

\section{Appendix A: Extensions of DATA}

Assume that we are given the set $F=N \backslash M$ and the metric $\left.g\right|_{F}$, but instead of the function $D_{x}: F \times F \rightarrow \mathbb{R}$ we know only its restriction on the boundary $\partial F=\partial M$, that is, the map

$$
\left.D_{x}\right|_{\partial F \times \partial F}: \partial F \times \partial F \rightarrow \mathbb{R},\left.D_{x}\right|_{\partial F \times \partial F}\left(z_{1}, z_{2}\right):=d_{N}\left(z_{1}, x\right)-d_{N}\left(z_{2}, x\right) .
$$

Lemma 3.2. The manifold $F=N \backslash M$, the metric $\left.g\right|_{F}$, and the restriction $\left.D_{x}\right|_{\partial F \times \partial F}$ of the distance difference function corresponding to $x \in M$ determine the distance difference function $D_{x}: F \times F \rightarrow \mathbb{R}$.

Proof. We can determine the map $D_{x}: F \times F \rightarrow \mathbb{R}$ by the formula

$$
D_{x}\left(z_{1}, z_{2}\right)=\inf _{\alpha} \sup _{\beta}\left(\mathcal{L}(\alpha)+\left.D_{x}\right|_{\partial F \times \partial F}(\alpha(1), \beta(1))-\mathcal{L}(\beta)\right),
$$

where the infimum is taken over the smooth curves $\alpha:[0,1] \rightarrow F$ from $z_{1}$ to $\alpha(1) \in \partial F$ and the supremum is taken over the smooth curves $\beta:[0,1] \rightarrow F$ from $z_{2}$ to $\beta(1) \in \partial F$.

This raises the question, if the manifold $(N, g)$ can be reconstructed when we are given a submanifold of codimension 1 , e.g. the boundary of the open set $M$ considered above, and the distance difference functions 
on this submanifold. To consider this, assume that we are given a submanifold $\widetilde{F} \subset N$ of dimension $(n-1)$, the metric $\left.g\right|_{\widetilde{F}}$ on $\widetilde{F}$, and the collection

$$
\left\{D_{\widetilde{F}, N}^{x} ; x \in N\right\} \subset C(\widetilde{F} \times \widetilde{F}),
$$

where $D_{\widetilde{F}, N}^{x}\left(z_{1}, z_{2}\right)=d_{N}\left(x, z_{1}\right)-d_{N}\left(x, z_{2}\right)$ for $z_{1}, z_{2} \in \widetilde{F}$. The following counterexample shows that such data do not uniquely determine the isometry type of $(N, g)$.

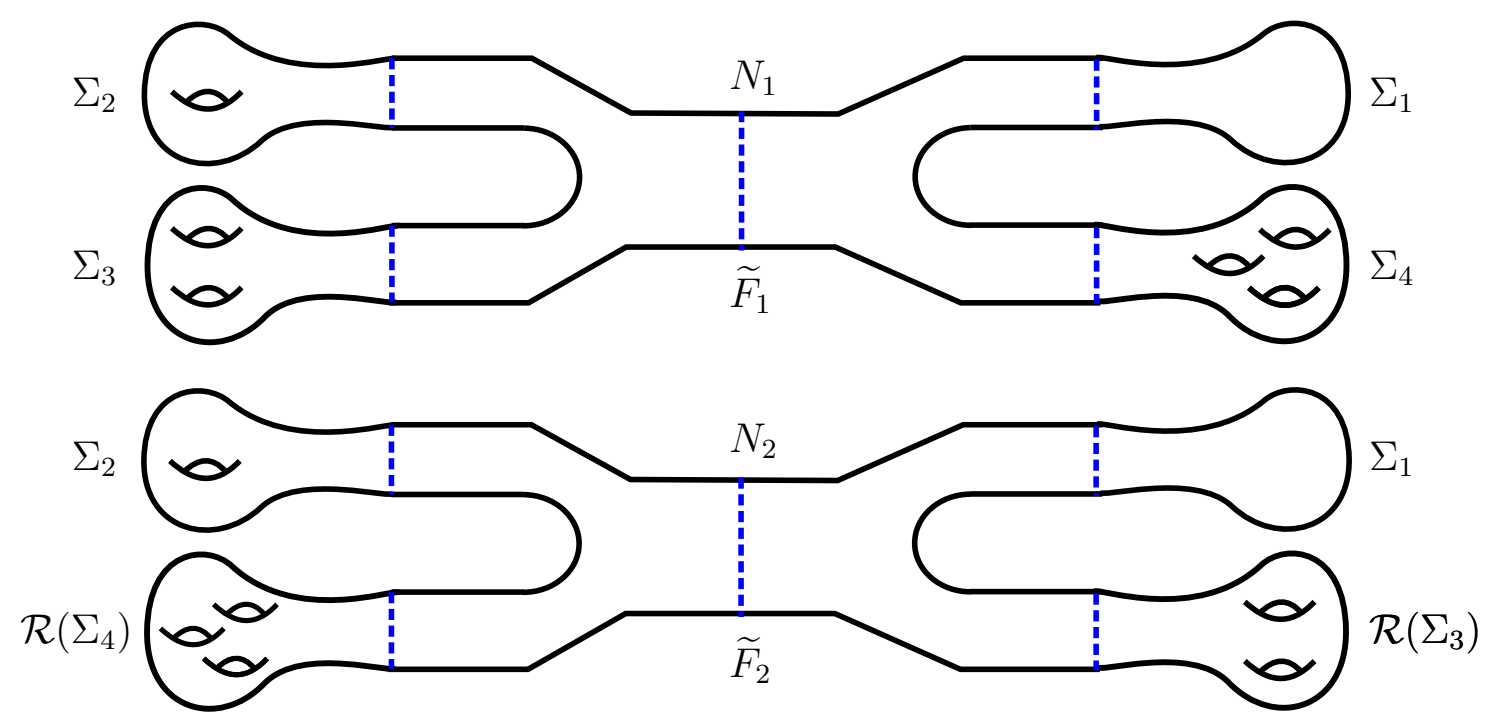

Figure 5. An illustration of manifolds $N_{1}$ and $N_{2}$ in Example $A 1$. When $(n-1)$-dimensional submanifolds $\widetilde{F}_{1}=\widetilde{F}_{2}=\widetilde{F}$ are identified, the distance difference functions $\left\{D_{\widetilde{F}, N_{1}}^{x} ; x \in N_{1}\right\}$ and $\left\{D_{\widetilde{F}, N_{2}}^{x} ; x \in N_{2}\right\}$ coincide.

Example A1. Let $C_{r}(y)=\left\{\left(x_{1}, x_{2}\right) \in \mathbb{R}^{2} ;\left|x_{1}-y_{1}\right|^{2}+\left|x_{2}-y_{2}\right|^{2}=r^{2}\right\}$ be a circle of radius $r$ centered at $y=\left(y_{1}, y_{2}\right)$. Let $p_{1}=(2,0), p_{2}=$ $(-2,0), L>3$, and

$$
\begin{aligned}
& S_{0}=C_{1}(0) \times[-1,1], \\
& S_{1}=C_{1}\left(p_{1}\right) \times[2, L], \\
& S_{2}=C_{1}\left(p_{2}\right) \times[2, L],
\end{aligned}
$$

and $K \subset \mathbb{R}^{2} \times[1,2]$ be a 2-dimensional surface which boundary has three components, $C_{1}(0) \times\{1\}, C_{1}\left(p_{1}\right) \times\{2\}$, and $C_{1}\left(p_{2}\right) \times\{2\}$, such that the union $S_{0} \cup K \cup S_{1} \cup S_{2}$ is a smooth surface in $\mathbb{R}^{3}$. Moreover, let $\mathcal{R}:\left(x_{1}, x_{2}, x_{3}\right) \mapsto\left(x_{1}, x_{2},-x_{3}\right)$ denote the reflection in the $x_{3}$-variable. Observe that then $\mathcal{R}\left(S_{0}\right)=S_{0}$. We define a smooth surface

$$
\Sigma_{0}=S_{0} \cup K \cup S_{1} \cup S_{2} \cup \mathcal{R}(K) \cup \mathcal{R}\left(S_{1}\right) \cup \mathcal{R}\left(S_{2}\right) .
$$


The boundary of $\Sigma_{0}$ consists of 4 circles, namely $\Gamma_{1}=C_{1}\left(p_{1}\right) \times\{L\}$, $\Gamma_{2}=C_{1}\left(p_{1}\right) \times\{-L\}, \Gamma_{3}=C_{1}\left(p_{2}\right) \times\{L\}$, and $\Gamma_{4}=C_{1}\left(p_{2}\right) \times\{-L\}$. Let us consider four embedded Riemannian surfaces $\Sigma_{j} \subset \mathbb{R}^{3}, j=1,2,3,4$, with boundaries $\partial \Sigma_{j}$ are equal to $\Gamma_{j}$. Assume that near $\partial \Sigma_{j}$ the surfaces $\Sigma_{j}$ are isometric to the Cartesian product of $\Gamma_{j}$ and an interval $[0, \varepsilon]$ with $\varepsilon>0$, and that the genus of $\Sigma_{j}$ is equal to $(j-1)$. Also, assume that $\Sigma_{j} \cap \Sigma_{k}=\emptyset$ for $j, k=1,2,3,4$ and $\Sigma_{0} \cap \Sigma_{j}=\Gamma_{j}$ for $j=1,2,3,4$.

First, let us construct a manifold $N_{1}$ by gluing surfaces $\Sigma_{0}$ with $\Sigma_{1}, \Sigma_{2}, \Sigma_{3}$, and $\Sigma_{4}$ such that the boundaries $\Gamma_{j}$ are glued with $\partial \Sigma_{j}$, $j \in\{1,2,3,4\}$.

Second, we construct a manifold $N_{2}$ by gluing surfaces $\Sigma_{0}$ with $\Sigma_{1}, \Sigma_{2}, \mathcal{R}\left(\Sigma_{3}\right)$, and $\mathcal{R}\left(\Sigma_{4}\right)$ such that the boundaries $\Gamma_{j}$ are glued with $\partial \Sigma_{j}$ with $j \in\{1,2\}$ but $\Gamma_{3}$ is glued with $\mathcal{R}\left(\partial \Sigma_{4}\right)$ and $\Gamma_{4}$ is glued with $\mathcal{R}\left(\partial \Sigma_{3}\right)$, see Fig. 5 . For both manifolds $N_{1}$ and $N_{2}$ we give the induced Riemannian metric from $\mathbb{R}^{3}$. Let $\widetilde{F}=\widetilde{F}_{1}=\widetilde{F}_{2}=S_{0} \cap\left(\mathbb{R}^{2} \times\{0\}\right)$.

Let us assume that $L$ above is larger than diam $(K)+10$. Then on $N_{\ell}, \ell=1,2$ a minimizing geodesic from $x \in \Sigma_{j}, j \geq 1$ to $z \in \widetilde{F}$ does not intersect the other sets $\Sigma_{k}$ with $k \in\{1,2,3,4\} \backslash\{j\}$. Using this we see that the sets $\left\{D_{\widetilde{F}, N_{\ell}}^{x} ; x \in N_{\ell}\right\} \subset C(\widetilde{F} \times \widetilde{F})$ are the same for $\ell=1,2$. As the manifolds $N_{1}$ and $N_{2}$ are not isometric, this implies that the data $\left(\widetilde{F},\left.g\right|_{\widetilde{F}}\right)$ and $\left\{D_{\widetilde{F}, N}^{x} ; x \in N\right\}$ do not determine uniquely the manifold $(N, g)$.

\section{REFERENCES}

[1] H. Ammari, E. Bossy, V. Jugnon, H. Kang: Mathematical modeling in photoacoustic imaging of small absorbers, SIAM Rev. 52 (2010), 677-695.

[2] M. Anderson, A. Katsuda, Y. Kurylev, M. Lassas, M. Taylor: Boundary regularity for the Ricci equation, Geometric Convergence, and Gel'fand's Inverse Boundary Problem, Inventiones Mathematicae 158 (2004), 261-321.

[3] B. Artman, I. Podladtchikov, B. Witten: Source location using timereverse imaging. Geophysical Prospecting 58(2010), 861-873.

[4] J. Bercoff, M. Tanter, M. Fink: Supersonic shear imaging: a new technique for soft tissue elasticity mapping, IEEE Trans. Ultrason. Ferroelectr. Freq. Control, 51 (2004), 396-409.

[5] G. Bal, T. Zhou: Hybrid inverse problems for a system of Maxwell's equations, Inverse Problems 30 (2014), no. 5, 055013, 17.

[6] G. Bal, G. Uhlmann: Inverse diffusion theory of photoacoustics. Inverse Problems 26 (2010), no. 8, 085010, 20.

[7] G. Bal: Hybrid inverse problems and internal functionals, Inside Out II, MSRI Publications, Ed. by G. Uhlmann, Cambridge Univ., (2012)

[8] G. Bal, R.Kui: Multi-source quantitative photoacoustic tomography in a diffusive regime. Inverse Problems 27 (2011), no. 7, 075003, 20.

[9] M. Belishev, Y. Kurylev: To the reconstruction of a Riemannian manifold via its spectral data (BC-method). Comm. PDE 17 (1992), 767-804.

[10] P. Berard, G. Besson, S. Gallot: Embedding Riemannian manifolds by their heat kernel. Geom. Funct. Anal. 4 (1994), 373-398 
[11] A. Bolsinov, V. Matveev: Geometrical interpretation of Benenti systems, J. Geom. Phys. 44(2003), 489-506.

[12] I. Chavel: Riemannian geometry A Modern Introduction, 2nd Edition, Cambridge Univ. (2006)

[13] M. Choulli, P. Stefanov: Inverse scattering and inverse boundary value problems for the linear Boltzmann equation. Comm. PDE 21 (1996), 763-785.

[14] M. de Hoop, S. Holman, E. Iversen, M. Lassas, B. Ursin: Recovering the isometry type of a Riemannian manifold from local boundary diffraction travel times, To appear in Journal de Mathématiques Pures et Appliquées.

[15] M. de Hoop, S. Holman, E. Iversen, M. Lassas, B. Ursin: Reconstruction of a conformally Euclidean metric from local boundary diffraction travel times, SIAM Journal on Applied Mathematics 46 (2014), 3705-3726.

[16] M. de Hoop, J. Tittelfitz: An inverse source problem for a variable speed wave equation with discrete-in-time sources. Inverse Problems.

[17] H. Isozaki, Y. Kurylev, M. Lassas: Conic singularities, generalized scattering matrix, and inverse scattering on asymptotically hyperbolic surfaces. J. reine angew. Math.

[18] J. Duistermaat, L. Hörmander: Fourier integral operators. II. Acta Math. 128 (1972), 183-269.

[19] A. Greenleaf, G., Uhlmann: Recovering singularities of a potential from singularities of scattering data. Comm. Math. Phys. 157 (1993), 549-572.

[20] S. Helgason: Differential geometry and symmetric spaces, Elsevier, (1962)

[21] L. Hörmander: The Analysis of Linear Partial Differential Operators IV: Fourier Integral Operators, Springer-Verlag Berlin Heidelberg, 2009

[22] P. Hoskins: Principles of ultrasound elastography, Ultrasound 20 (2012), 8-15.

[23] W. Jeong, H. Lim, H. Lee, J. Jo, Y. Kim: Principles and clinical application of ultrasound elastography for diffuse liver disease, Ultrasonography 33 (2014), 149-160.

[24] H. Kao, S.-J. Shan. The source-scanning algorithm: Mapping the distribution of seismic sources in time and space. Geophysical Journal International, 157 (2004), 589-594.

[25] J. Kayal: Microearthquake Seismology and Seismotectonics of South Asia, Springer, 2008, $552 \mathrm{pp}$.

[26] A. Katchalov, Y. Kurylev, Multidimensional inverse problem with incomplete boundary spectral data. Comm. PDE 23 (1998), 55-95.

[27] A. Katchalov, Y. Kurylev, M. Lassas: Inverse boundary spectral problems, Chapman and Hall (2001).

[28] W. Klingenberg: Riemannian geometry, Walter De Gruyter, 1982.

[29] K. Krupchyk, Y. Kurylev, M. Lassas: Inverse spectral problems on a closed manifold. J. Math. Pures et Appl. 90 (2008), 42-59.

[30] Y. Kurylev: Multidimensional Gel'fand inverse problem and boundary distance map, Inverse Problems Related with Geometry, Ed. H. Soga (1997), 1-15.

[31] Y. Kurylev, M. Lassas: Inverse Problems and Index Formulae for Dirac Operators. Adv. Math. 221 (2009), 170-216.

[32] Y. Kurylev, M. Lassas, E. Somersalo: Maxwell's equations with a polarization independent wave velocity: Direct and inverse problems, J. Math. Pures et Appl. 86 (2006), 237-270.

[33] Y. Kurylev, M. Lassas, G. Uhlmann: Rigidity of broken geodesic flow and inverse problems, Amer. J. Math. 132 (2010), 529-562.

[34] Y. Kurylev, M. Lassas, G. Uhlmann: Seeing through spacetime, 67 pp. ArXiv:1405.3386. 
[35] Y. Kurylev, L. Oksanen, G. Paternain: Inverse problems for the connection Laplacian. ArXiv:1509.02645.

[36] M. Lassas, L. Oksanen: Inverse problem for the Riemannian wave equation with Dirichlet data and Neumann data on disjoint sets. Duke Math. J. 163 (2014), 1071-1103.

[37] M. Lassas, T. Saksala: Determination of a Riemannian manifold from the distance difference functions with an appendix on Matveev-Topalov theorem. (An extended preprint version of this paper), see http://wiki.helsinki.fi/ display/mathstatHenkilokunta/Teemu+Saksal

[38] T. Levi-Civita: Sulle trasformazioni delle equazioni dinamiche, Ann. di Mat., 24(1896), 255-300.

[39] W. Ledermann: A note on skew symmetric determinants, Proc. Edinburgh Math. Soc. 36 (1993), 335-338.

[40] J. Lee: Introduction to smooth manifolds, Springer (2000)

[41] J. Lee: Riemannian manifolds An Introduction to Curvature, Springer (1997)

[42] H. Liu, G. Uhlmann: Determining both sound speed and internal source in thermo- and photo-acoustic tomography, Inverse Problems 31 (2015), 105005

[43] V. Matveev, P. Topalov: Geodesic Equivalence via Integrability, Geometriae Dedicata 96 (2003), 91-115.

[44] V. Matveev, P. Topalov: Trajectory equivalence and corresponding integrals, Regular Chaotic Dynam. 3(2) (1998), 30-45.

[45] V. Matveev, P. Topalov: Quantum integrability for the Beltrami-Laplace operator as geodesic equivalence, Math. Z. 238 (2001), 833-866.

[46] V. Matveev: Geodesically equivalent metrics in general relativity, J. Geom. and Phys. 62 (2012), 675-691.

[47] S. McDowall, P. Stefanov, A. Tamasan: Gauge equivalence in stationary radiative transport through media with varying index of refraction, Inverse Problems and Imaging 4 (2010), 151-168.

[48] S. McDowall: Optical tomography on simple Riemannian surfaces, Comm. PDE. 30 (2005), 1379-1400.

[49] S. McDowall: An inverse problem for the transport equation in the presence of a Riemannian metric, Pac. J. Math 216 (2004), 107-129.

[50] B. O'Neill, Semi-Riemannian geometry. With applications to relativity. Pure and Applied Mathematics, 103. Academic Press, Inc., 1983. xiii +468 pp.

[51] L. Oksanen: Solving an inverse problem for the wave equation by using a minimization algorithm and time-reversed measurements, Inverse Probl. Imaging 5 (2011), 731-744.

[52] L. Oksanen: Solving an inverse obstacle problem for the wave equation by using the boundary control method, Inverse Problems 29 (2013), 035004.

[53] J. Ophir et al.: Elastography: ultrasonic estimation and imaging of the elastic properties of tissues. Proc. Inst. Mech. Eng. H. 213 (1999), 203-233.

[54] L. Patrolia: Quantitative photoacoustic tomography with variable index of refraction. Inverse Probl. Imaging 7 (2013), 253-265.

[55] L. Pestov, G. Uhlmann, H. Zhou: An inverse kinematic problem with internal sources, Inverse Problems 31, 055006 (2015), 6.

[56] P. Petersen, Riemannian geometry. Springer, 1998. xvi+432 pp.

[57] J. Qian, P. Stefanov, , G. Uhlmann and H. Zhao: An efficient Neumann seriesbased algorithm for thermoacoustic and photoacoustic tomography with variable sound speed, SIAM J. Imaging Sci. 4(3) (2011), 850-883 pp

[58] P. Sava, Micro-earthquake monitoring with sparsely-sampled data, Journal of Petroleum Exploration and Production Technology 1 (2011), 43-49. 
[59] P. Stefanov, G. Uhlmann: Instability of the linearized problem in multiwave tomography of recovery both the source and the speed, Inverse Probl. and Imaging, 7(4) (2013), 1367-1377.

[60] P. Stefanov, G. Uhlmann: Recovery of a Source or a Speed with One Measurement and Applications, Transactions of AMS 365 (2013), 5737-5758.

[61] P. Stefanov, G. Uhlmann: Multi-wave methods via ultrasound. Inside Out II, MSRI publications, 60:271-324, 2012.

[62] P. Stefanov, G. Uhlmann: Thermoacoustic tomography with variable sound speed, Inverse Problems 25 (2009), 075011.

[63] P. Topalov: Geodesic compatibility and integrability of geodesic flows, J. Math. Phys. 44 (2003), 913-929,

Matti Lassas and Teemu Saksala, Department of Mathematics and Statistics, University of Helsinki, Finland 